\title{
Primordial Compositions of Refractory Inclusions
}

L. Grossman, S. B. Simon, V. K. Rai, M. H. Thiemens, I. D. Hutcheon, R. W. Williams, A. Galy, T. Ding, A. V. Fedkin, R. N. Clayton, T. K. Mayeda

February 25, 2008

Geochimica et Cosmochimica Acta 
This document was prepared as an account of work sponsored by an agency of the United States government. Neither the United States government nor Lawrence Livermore National Security, LLC, nor any of their employees makes any warranty, expressed or implied, or assumes any legal liability or responsibility for the accuracy, completeness, or usefulness of any information, apparatus, product, or process disclosed, or represents that its use would not infringe privately owned rights. Reference herein to any specific commercial product, process, or service by trade name, trademark, manufacturer, or otherwise does not necessarily constitute or imply its endorsement, recommendation, or favoring by the United States government or Lawrence Livermore National Security, LLC. The views and opinions of authors expressed herein do not necessarily state or reflect those of the United States government or Lawrence Livermore National Security, LLC, and shall not be used for advertising or product endorsement purposes. 


\title{
Primordial Compositions of Refractory Inclusions
}

\author{
L. Grossman ${ }^{1}$, S. B. Simon \\ Dept. of the Geophysical Sciences \\ The University of Chicago \\ Chicago, Illinois 60637, U.S.A. \\ V. K. Rai ${ }^{2}$, M. H. Thiemens \\ Dept. of Chemistry and Biochemistry \\ University of California \\ San Diego, California 92093-0352, U.S.A. \\ I. D. Hutcheon, R. W. Williams \\ Lawrence Livermore National Laboratory \\ Livermore, California 94551-0808, U.S.A.
}

\author{
A. Galy \\ Dept. of Earth Science \\ University of Cambridge \\ Cambridge, CB2 3EQ England. \\ T. Ding \\ Institute of Mineral Resources \\ Chinese Academy of Geological Sciences \\ Beijing 100037, China \\ A. V. Fedkin, R. N. Clayton ${ }^{1}$ \\ Dept. of the Geophysical Sciences \\ The University of Chicago \\ Chicago, Illinois 60637, U.S.A
T. K. Mayeda ${ }^{3}$
Enrico Fermi Institute
The University of Chicago
Chicago, Illinois 60637 \\ Submitted to Geochimica et Cosmochimica Acta \\ November 27, 2007.
}

\footnotetext{
${ }^{1}$ Also Enrico Fermi Institute, The University of Chicago.

${ }^{2}$ Present address: Planetary and Geosciences Division, Physical Research Laboratory, Navarangpura, Ahmedabad 380 009, India.

${ }^{3}$ Deceased.
} 


\begin{abstract}
Bulk chemical and oxygen, magnesium and silicon isotopic compositions were measured for each of 17 Types A and B refractory inclusions from CV3 chondrites. After bulk chemical compositions were corrected for non-representative sampling in the laboratory, the $\mathrm{Mg}$ and $\mathrm{Si}$ isotopic compositions of each inclusion were used to calculate its original chemical composition assuming that the heavy-isotope enrichments of these elements are due to Rayleigh fractionation that accompanied their evaporation from CMAS liquids. The resulting pre-evaporation chemical compositions are consistent with those predicted by equilibrium thermodynamic calculations for high-temperature nebular condensates but only if different inclusions condensed from nebular regions that ranged in total pressure from $10^{-6}$ to $10^{-1}$ bar, regardless of whether they formed in a system of solar composition or in one enriched in OC dust relative to gas by a factor of ten relative to solar composition. This is similar to the range of total pressures predicted by dynamic models of the solar nebula for regions whose temperatures are in the range of silicate condensation temperatures. Alternatively, if departure from equilibrium condensation and/or non-representative sampling of condensates in the nebula occurred, the inferred range of total pressure could be smaller. Simple kinetic modeling of evaporation successfully reproduces observed chemical compositions of most inclusions from their inferred pre-evaporation compositions, suggesting that closed-system isotopic exchange processes did not have a significant effect on their isotopic compositions. Comparison of pre-evaporation compositions with observed ones indicates that $80 \%$ of the enrichment in refractory $\mathrm{CaO}+\mathrm{Al}_{2} \mathrm{O}_{3}$ relative to more volatile $\mathrm{MgO}+\mathrm{SiO}_{2}$ is due to initial condensation and $20 \%$ due to subsequent evaporation for both Type A and Type B inclusions.
\end{abstract}

\title{
1. INTRODUCTION
}

Whether calcium-, aluminum-rich inclusions (CAIs) in CV3 chondrites are solar nebular condensates or evaporation residues has been debated since the earliest days of research on these objects, with Christophe Michel-Levy (1968) and Grossman (1972) suggesting that they are condensates and Chou et al. (1976) and Notsu et al. (1978) arguing that they are residues. Grossman et al. (2000) seemed to provide the answer to this important question when they argued that they are actually both. They noted that major element bulk compositions of coarse-grained inclusions, when corrected for nonrepresentative sampling in the laboratory, are deficient in $\mathrm{MgO}$ and $\mathrm{SiO}_{2}$ compared to those predicted by thermodynamic calculations for high-temperature, solar nebular condensates, and that enrichments in the heavy isotopes of $\mathrm{Mg}$ and $\mathrm{Si}$ reported for such inclusions, when interpreted as being due to partial evaporation of these oxides, can 
account approximately for the magnitudes of these deficits. Thus, Grossman et al. (2000) concluded that the major element compositions of these objects resulted from hightemperature condensation of refractory assemblages, followed by evaporation of several tens of percent of the $\mathrm{Mg}$ and $\mathrm{Si}$ from them during partial melting in a hydrogen-rich gas.

In that work, the chemical compositions were determined for one group of inclusions, and the isotopic compositions for another. If, instead, both the present chemical composition and the $\mathrm{Mg}$ and $\mathrm{Si}$ isotopic compositions were known for any one inclusion, the bulk composition of its precursor could be determined by peeling away the effects of evaporation. Furthermore, if the precursor compositions were known for each of a suite of inclusions, comparisons with predictions of condensation models could be used to draw inferences about how representatively each object sampled solar nebular condensates, the total pressure and dust/gas ratio of the system from which the inclusions condensed and the temperatures at which they became chemically isolated from the gas. This paper presents the results of such a study. Preliminary versions of this work were given by Simon et al. (2004) and Grossman et al. (2007b).

\section{ANALYTICAL TECHNIQUES}

\subsection{Sampling}

Relatively large, coarse-grained, white inclusions were located on slab surfaces of the Allende, Efremovka, Leoville and Vigarano CV3 chondrites. Those selected ranged from two $\mathrm{mm}$ to more than one $\mathrm{cm}$ in shortest dimension, and, based on their rounded outlines, appeared to be complete, unfragmented individuals. Material was excavated from each one using stainless steel dental tools, and ground into a powder with an agate mortar and pestle. For the present work, each powdered sample, weighing from 5 to 200 $\mathrm{mg}$ but most $\sim 40 \mathrm{mg}$, was separated into aliquots for major element chemical analysis, magnesium isotopic analysis, and oxygen and silicon isotopic analysis. Aliquots of two of the samples were also used for U-Pb age determinations by Amelin et al. (2002). In most, but not all, cases, the mirror image of each inclusion on a facing slab was made into one or more polished thin sections.

\subsection{Major element chemical analysis}

A bulk chemical analysis was obtained for each sample by modal recombination of wavelength-dispersive electron microprobe (EPMA) spot analyses of mineral phases in the thin section(s) or by instrumental neutron activation analysis (INAA) of one of the aliquots or by both methods. Details of the techniques employed and of our reasons for preferring modal recombination data were given in Simon and Grossman (2004). 


\subsection{Magnesium isotopic analysis}

At Lawrence Livermore National Laboratory (LLNL), samples were dissolved in an $\mathrm{HNO}_{3}-\mathrm{HF}-\mathrm{HClO}_{4}$ acid solution at $\sim 100^{\circ} \mathrm{C}$ in sealed PFA Teflon vials, taken to dryness and then digested a second time in an $\mathrm{HCl}-\mathrm{HF}-\mathrm{HClO}_{4}$ acid solution. The samples were dried again, converted to nitrate with $\mathrm{HNO}_{3}$ and finally redissolved in $1 \mathrm{M}$ $\mathrm{HNO}_{3}$. Insoluble residues (spinel + perovskite) were separated by centrifugation. $\mathrm{Mg}$ was purified from the dissolved fraction on AG50W-X8 (200-400 mesh) resin columns with quantitative recovery. The $\mathrm{Mg}$ isotope compositions were measured with the LLNL IsoProbe multi-collector, magnetic sector ICP-MS, using static multi-collection. $\mathrm{Mg}$ isotope compositions are reported as $\delta^{25} \mathrm{Mg}$ and $\delta^{26} \mathrm{Mg}$, determined with the standardsample-standard bracketing technique and calculated relative to the DSM3 Mg metal standard (Galy et al., 2003). The purified Mg from each CAI was analyzed at least 3 times, and the uncertainties quoted are two standard deviations of the mean. The average reproducibility for these measurements is $0.15 \%$ and $0.40 \%$ for $\delta^{25} \mathrm{Mg}$ and $\delta^{26} \mathrm{Mg}$, respectively.

At Cambridge University, methods of chemical digestion, $\mathrm{Mg}$ separation and measurement of $\mathrm{Mg}$ isotopic composition were similar to those used by Galy et al. (2000). The samples were powdered and digested in sealed PFA Teflon ${ }^{\circledR}$ vials at $\sim 140^{\circ} \mathrm{C}$ with $0.5 \mathrm{ml}$ distilled $\mathrm{HNO}_{3}$ and $0.5 \mathrm{ml}$ distilled $\mathrm{HF}$. After removal of silicon by evaporation and conversion of nitrate salts into chloride salts, $\mathrm{Al}$ and Fe were removed by co-precipitation with $\mathrm{NH}_{3}$ at $\mathrm{pH} \leq 7$. Mg was then separated from $\mathrm{Ca}$ and other elements using Biorad AG50 X12 cation exchange resin. Quantitative recovery of Mg has been demonstrated both by ICP AES and by purifying a multi-element solution with known

$\mathrm{Mg}$ isotope ratios (Tipper et al., 2006). Typically, $20 \mu \mathrm{g}$ of $\mathrm{Mg}$ are processed. The procedural blanks for $\mathrm{Mg}$ were $<1 \mathrm{ng}$, less than 0.001 of the total $\mathrm{Mg}$. Following chemical separation, all samples were dissolved in $0.3 \mathrm{~N} \mathrm{HNO}_{3}$ and centrifuged before introduction into the mass spectrometer. High-precision $\mathrm{Mg}$ isotopic abundances were obtained with a Nu-instruments MC-ICPMS (Galy et al., 2001). All samples and standards were introduced into the plasma torch through an Aridus desolvating nebulizer. Use of 3 Faraday collectors over a mass range of 24 to 26 allowed direct measurement of ${ }^{24} \mathrm{Mg},{ }^{25} \mathrm{Mg}$ and ${ }^{26} \mathrm{Mg}$. Standard and sample were measured alternately for $200 \mathrm{~s}$ each. Negligible cross-contamination between sample and standard was achieved by washing the analytical instrumentation system and the cones with $0.1 \mathrm{~N} \mathrm{HNO}_{3}$ for 5 min between analyses. Each sample underwent at least duplicate mass spectrometric analysis. An estimate of the long-term total procedural precision and accuracy on $\delta^{25} \mathrm{Mg}$ obtained by measurement of a multi-element standard solution is $0.07 \%$ (Tipper et al., 2006).

\subsection{Oxygen and silicon isotopic analysis}


At the University of Chicago, reaction of silicates and oxides with bromine pentafluoride $\left(\mathrm{BrF}_{5}\right)$ gave quantitative yields of both oxygen $\left(\mathrm{as}_{2}\right)$ and silicon $\left(\mathrm{as} \mathrm{SiF}_{4}\right)$. Oxygen was purified using 13X molecular sieve as in Clayton and Mayeda (1983). $\mathrm{SiF}_{4}$ was purified by gas chromatography as in Molini-Velsko et al. (1986). Measurements of $\delta^{18} \mathrm{O}, \delta^{17} \mathrm{O}, \delta^{30} \mathrm{Si}$ and $\delta^{29} \mathrm{Si}$ were done with double-collecting mass spectrometers. The $1 \sigma$ uncertainty on these data is $\pm 0.2 \%$.

Some of the fluorinations and oxygen isotopic analyses were performed at the University of California, San Diego using the following procedure. Nickel tubes were cleaned under high vacuum by heating and pumping at $400{ }^{\circ} \mathrm{C}$ overnight. A nickel tube was filled with dry nitrogen and placed in a dry box, together with a 1-3 mg powdered sample, where they were left for at least six hours before the tube was opened and the sample powder loaded into it. The tube was reconnected to the extraction line and heated under vacuum at $\sim 300{ }^{\circ} \mathrm{C}$ overnight. $\mathrm{BrF}_{5}$ was added to the reaction tube, kept at room temperature for six hours (in most cases) to remove adsorbed water, and pumped away. A new batch of $\mathrm{BrF}_{5}$ was cleaned of non-condensable impurities by freezing at $-90^{\circ} \mathrm{C}$, and then was added to the tube and heated to $700^{\circ} \mathrm{C}$ for $36 \mathrm{hr}$. The reaction products were passed through two liquid-nitrogen-cooled traps, and the $\mathrm{O}_{2}$ was collected by freezing onto a molecular sieve. The oxygen isotopic measurements were carried out on a Finnigan MAT-251 mass spectrometer. The cold-trap was warmed to $-120^{\circ} \mathrm{C}$ to evaporate $\mathrm{SiF}_{4}$, which was transferred into a quarter-inch Pyrex tube. All the sealed tubes of $\mathrm{SiF}_{4}$ were sent to the Institute of Mineral Resources of the Chinese Academy of Geological Sciences in Beijing for silicon isotopic measurements. Several NBS-28 samples were processed in the same way as a check on the procedure.

In Beijing, additional NBS-28 samples were fluorinated by the procedure of Clayton and Mayeda (1963), after first separating $\mathrm{CF}_{4}, \mathrm{SiF}_{4}$ and $\mathrm{SF}_{6}$ impurities from $\mathrm{BrF}_{5}$ obtained from the Wuhan Institute of Chemical Products. Because of the different vapor pressures of these species, the impurities can be removed by multiple cycles of cryogenic vaporization and condensation using an ethanol-liquid nitrogen mixture $\left(-70^{\circ} \mathrm{C}\right)$. At this temperature, $\mathrm{BrF}_{5}$ remains in the solid phase, but the impurities are present in the vapor phase and can be pumped to waste. A dry ice-acetone mixture $\left(-80^{\circ} \mathrm{C}\right)$ was used to extract the $\mathrm{SiF}_{4}$ fluorination product from the $\mathrm{BrF}_{5}$ reagent.

Silicon isotope measurements of samples and standards were carried out with a MAT-253 gas source isotope ratio mass spectrometer as described by Ding (2004) and Ding et al. (2005). A $9.12 \mathrm{kV}$ accelerating voltage was used with a magnetic field intensity of 0.9509 T. Masses 85,86 and 87 were collected simultaneously, and the results are expressed as $\delta^{29} \mathrm{Si}$ and $\delta^{30} \mathrm{Si}$ values relative to NBS-28. Six samples of NBS28 that were fluorinated in San Diego were isotopically analysed in Beijing against NBS28 that was fluorinated in Beijing. The mean $\delta^{29} \mathrm{Si}$ and $\delta^{30} \mathrm{Si}$ were 0.019 and $0.037 \%$, respectively. Their $1 \sigma$ standard deviations were \pm 0.092 and \pm 0.177 , respectively, which 
include uncertainty due to blanks, fluorination, extraction and measurement. Thus, $1 \sigma$ uncertainties of \pm 0.1 for $\delta^{29} \mathrm{Si}$ and \pm 0.2 for $\delta^{30} \mathrm{Si}$ were adopted for the meteorite samples.

\section{RESULTS}

\subsection{Major element bulk compositions}

As defined by Grossman (1980), coarse-grained, Ca-, Al-rich inclusions in CV3 chondrites are found in two mineralogical varieties. The primary phase assemblage of Type As consists of melilite (a solid solution between gehlenite $\left(\mathrm{Ca}_{2} \mathrm{Al}_{2} \mathrm{SiO}_{7}\right)$ and åkermanite $\left(\mathrm{Ca}_{2} \mathrm{MgSi}_{2} \mathrm{O}_{7}\right)$, spinel $\left(\mathrm{MgAl}_{2} \mathrm{O}_{4}\right)$, perovskite $\left(\mathrm{CaTiO}_{3}\right)$ and $<35$ vol\% fassaite, a Ca-, Al-rich clinopyroxene rich in diopside $\left(\mathrm{CaMgSi}_{2} \mathrm{O}_{6}\right)$ and containing both

$\mathrm{Ti}^{3+}$ and $\mathrm{Ti}^{4+}$ (Dowty and Clark, 1973). These are further subdivided into "fluffy" and compact sub-types, the former much more irregularly-shaped and heavily altered than the latter. The primary phase assemblage of Type Bs consists of melilite, spinel, anorthite $\left(\mathrm{CaAl}_{2} \mathrm{Si}_{2} \mathrm{O}_{8}\right)$ and $\geq 35 \mathrm{vol} \%$ fassaite. These were subdivided by Wark and Lovering (1977) into B1s, which contain massive melilite mantles and B2s, which do not.

All of the major element chemical data used in the present study were given by Simon and Grossman (2004), except for those of the compact Type A (CTA) inclusions, F11, TS32, E49 and E56; the Type B inclusions, TS34 and 1623-8; and the unusual forsterite $\left(\mathrm{Mg}_{2} \mathrm{SiO}_{4}\right)$-bearing Type B, E60. All of these are listed in Table 1 except for 1623-8, for which the INAA data of Sylvester et al. (1992) were used. In the case of the INAA data for E60, the quoted errors on Table 1 are the $1 \sigma$ uncertainties due only to counting statistics. For all inclusions whose compositions were determined by INAA (E13, E49A, E55, E60, E62 and 1623-8), $\mathrm{SiO}_{2}$ was determined by difference. Consequently, the uncertainty in this oxide may be significantly underestimated by the quoted counting statistics due to the possible concentration of any systematic errors in determining all of the other elements. As found by Simon and Grossman (2004), the CTAs tend to have lower $\mathrm{MgO}$ and $\mathrm{SiO}_{2}$ contents than Type Bs. The CTA measurements in this study yield $\mathrm{MgO}$ and $\mathrm{SiO}_{2}$ contents between 3 and $6 \mathrm{wt} \%$ and between 21 and 26 wt $\%$, respectively, compared to 10.74 and $26.55 \mathrm{wt} \%$, respectively, in the single Type B analyzed here. The forsterite-bearing Type $\mathrm{B}, \mathrm{E} 60$, contains much more $\mathrm{MgO}$ and $\mathrm{SiO}_{2}$ than any Type A or B inclusion, consistent with its high modal abundance of forsterite.

\subsection{Magnesium isotopic compositions}

The Livermore and Cambridge measurements of $\delta^{26} \mathrm{Mg}$ and $\delta^{25} \mathrm{Mg}$, with their $2 \sigma$ uncertainties, are given in Table 2 for all samples in this study. The $\delta^{25} \mathrm{Mg}$ data from the two laboratories are plotted against one another in Figure 1, from which the excellent agreement between them is evident. Of the eight samples whose magnesium isotopic 
data were measured in both laboratories, only two deviate significantly from the 1:1 line, TS33 slightly so and TS32 much more so. These differences are probably due to internal sample heterogeneity.

\subsection{Oxygen isotopic compositions}

The Chicago and San Diego measurements of $\delta^{18} \mathrm{O}$ and $\delta^{17} \mathrm{O}$ are given in Table 2 for all samples in this study. The data are plotted on the standard $\delta^{17} \mathrm{O}$ vs $\delta^{18} \mathrm{O}$, threeisotope plot in Figure 2, along with the Terrestrial Fractionation (TF) line and the Carbonaceous Chondrite Anhydrous Mineral (CCAM) line, the latter defined by compositions of many bulk, anhydrous inclusions in carbonaceous chondrites and their mineral separates measured in Chicago (Clayton et al., 1977). Although the UCSD data scatter more widely about the CCAM line than the Chicago data, the points generally lie quite close to the CCAM line. The sample with the highest $\delta^{17} \mathrm{O}(-6.5 \%$ ) however, E49, lies to the ${ }^{18} \mathrm{O}$-rich side of the CCAM line, deviating from it by $1.7 \%$, measured in a direction parallel to the $\delta^{18} \mathrm{O}$ axis. A special group of CAIs, known as FUN inclusions, exhibit extraordinarily large isotopic mass-fractionations of several elements, as a consequence of which their bulk oxygen isotopic compositions plot very far away from the CCAM line, to the ${ }^{18} \mathrm{O}$-rich side. Of all known FUN inclusions, EK1-4-1 is the one with the least mass-fractionated oxygen (McKeegan and Leshin, 2001). If its bulk $\delta^{17} \mathrm{O}$ were the same as that of E49, the deviation of EK1-4-1 from the CCAM line would be $1.4 \%$, less than that of E49, making the latter a candidate for a FUN inclusion. Another characteristic of FUN inclusions, however, is their very low initial ${ }^{26} \mathrm{Al} /{ }^{27} \mathrm{Al}$ ratios, inferred from their very small to non-existent ${ }^{26} \mathrm{Mg}$ excesses (MacPherson et al., 2005). Table 2 shows that $\mathrm{E} 49$ has a ${ }^{26} \mathrm{Mg}$ excess of at least $0.7 \%$. Apparently, neither E49 nor any of the other inclusions in this study are FUN inclusions.

\subsection{Silicon isotopic compositions}

The Beijing measurements of $\delta^{30} \mathrm{Si}$ and $\delta^{29} \mathrm{Si}$, and Chicago results for $\delta^{30} \mathrm{Si}$, are given in Table 2. In Figure 3, $\delta^{29} \mathrm{Si}$ is plotted against $\delta^{30} \mathrm{Si}$ for all samples used in this study for which both were measured, i.e., the Beijing data and samples F11, TS34 and F7 from Molini-Velsko (1983). The line shown is the average of the two least-squares regression lines through the Beijing data. It has a slope of $0.500 \pm 0.001$ and an intercept of $0.065 \pm 0.003$, indicating that the isotopic variations are dominated by mass-dependent fractionation and that no significant impurities of interfering masses survived the chemical procedure for $\mathrm{SiF}_{4}$ separation. When the three data points from Molini-Velsko (1983) are included, the slope of the mean regression becomes $0.506 \pm 0.002$ and the intercept becomes $0.034 \pm 0.005$. 


\section{DISCUSSION}

\subsection{Major element bulk compositions corrected for sampling bias}

Grossman et al. (2000) stressed the difficulty of obtaining representative analyses of refractory inclusions when sampling small fractions of them, and attempted to overcome this laboratory bias. They did so by assuming that every Type A and B CAI has a chondritic $\mathrm{Ca} / \mathrm{Al}$ ratio, and correcting published major element compositions of a suite of these objects by adding or subtracting spinel until this ratio was achieved. This approach was modified by Simon and Grossman (2004), who calculated a range of possible compositions for each object by computing how much spinel, melilite and fassaite, taken one at a time, had to be added to or subtracted from the bulk composition measured by modal recombination, in order to achieve a chondritic $\mathrm{Ca} / \mathrm{Al}$ ratio. For each inclusion, a best estimate of the bulk composition was obtained by taking the mean of the separate corrected compositions.

Incorporated into the present work are six of the inclusions whose mean compositions were determined in this way by Simon and Grossman (2004). Added to these are another eleven inclusions: four whose chemical compositions were also reported by Simon and Grossman (2004) but which were measured by INAA, six whose chemical compositions were determined since that work and are shown in Table 1, and another, 1623-8, whose composition was taken from the literature. Among these additional eleven inclusions are five whose chemical compositions were determined by modal recombination and thus could be corrected for non-representative sampling as in Simon and Grossman (2004).

Four of the remaining six inclusions are compact Type As, and E60 is a forsteritebearing Type B. Because thin sections of these samples were not available, their chemical compositions were obtained by INAA. Melilite and fassaite are important phases in compact Type As, and each exhibits a wide range of solid solution compositions within a given inclusion. Because the modal recombination technique makes use of large numbers of analyses of each of these phases within each inclusion, corrected bulk compositions are based on very good estimates of the mean compositions of melilite and fassaite. For each of these minerals, the grand average was taken of the average composition obtained for each compact Type A inclusion, and is listed in Table 3. For those compact Type A inclusions whose compositions were measured by INAA, the data in Table 3 were used to correct for non-representative sampling. Because the sampling corrections are not based on mineral analyses from the same inclusions, this procedure may introduce yet another source of uncertainty beyond that expressed by the formal error bars for those inclusions whose compositions were measured by INAA. Since the average melilite and fassaite compositions are not well-known for forsteritebearing CAIs, the sampling correction for E60 was based only on spinel. In the case of 
1623-8, the sixth CAI for which a composition determination by INAA was used here, another estimate of its chemical composition made by modal recombination (MacPherson and Davis, 1993) gave grossly different results, and was rejected. Nevertheless, mineral compositions from the same thin section used for the modal recombination were used for the sampling corrections to the INAA data.

The best estimate of the major element composition of each of the inclusions investigated in this work, after re-normalization to $100 \% \mathrm{CaO}+\mathrm{MgO}+\mathrm{Al}_{2} \mathrm{O}_{3}+\mathrm{SiO}_{2}$ (CMAS), is listed on Table 4.

\subsection{Condensation paths for solar composition}

Equilibrium condensation calculations were performed at $2 \mathrm{~K}$ temperature intervals at discrete total pressures between $10^{-1}$ and $5 \times 10^{-7}$ bar, using the VAPORS program of Ebel and Grossman (2000), the thermodynamic data and solution models therein, and updated solar system abundance data for $\mathrm{O}, \mathrm{C}$ and $\mathrm{S}$ that were discussed and employed by Fedkin and Grossman (2006). The bulk chemical composition of the $\mathrm{TiO}_{2}$ and metal-free condensate assemblage was calculated at each temperature step. "Condensation paths" are simply curves connecting bulk chemical compositions calculated at successive temperature steps. Over the temperature range where calculated bulk compositions are closest to those observed for CAIs, a condensation path for $\mathrm{MgO}$ and $\mathrm{SiO}_{2}$ is shown in Figure 4a for each of several different, relatively low values of total pressure, $P^{\text {tot }}$. Because of significant overlaps, paths for relatively high $P^{\text {tot }}$ are shown in a separate figure, $4 \mathrm{~b}$, for clarity. Because $\mathrm{Ca}$ and $\mathrm{Al}$ are always totally condensed above the temperature at which $\mathrm{MgO}$ reaches $1 \mathrm{wt} \%$ of the condensate assemblage, all calculated condensate compositions plotted in these figures have the chondritic $\mathrm{Ca} / \mathrm{Al}$ ratio. The paths in Figures $4 \mathrm{a}$ and $4 \mathrm{~b}$ differ from those in Grossman et al. (2000) because of the generally finer temperature resolution and updated abundance data employed herein. In particular, because the $\mathrm{C} / \mathrm{O}$ ratio is $19 \%$ greater in this work than in the Anders and Grevesse (1989) abundance table used by Grossman et al. (2000), there is less free oxygen available for oxide and silicate condensates, resulting in slightly lower condensation temperatures in this work, and some changes in condensation sequences.

Discontinuities break each condensation path into segments at temperatures where new phases condense and/or previously condensed phases disappear. Many of the segments in these figures have labels. These are the stable condensate phase assemblages whose bulk compositions are plotted along the segments. It is seen that there are ranges of $P^{\text {tot }}$ over which the sequence of condensate assemblages and, consequently, over which the condensation paths are quite similar to one another. Condensation paths may be quite different from one pressure range to another, however. For all $P^{\text {tot }} \geq 1 \times 10^{-5}$ bar, there is a segment from 7 to $10 \mathrm{wt} \% \mathrm{MgO}$ and $18-20 \mathrm{wt} \% \mathrm{SiO}_{2}$ over which the stable condensates are melilite + spinel + perovskite, the characteristic assemblage of Type A 
inclusions. The corresponding assemblage at lower $P^{\text {tot }}$ is melilite + spinel + fassaitic clinopyroxene. As temperature falls, melilite in these assemblages is calculated to react with gaseous $\mathrm{Si}$ and $\mathrm{Mg}$ to form clinopyroxene at $3 \times 10^{-3} \geq P^{t o t} \geq 2 \times 10^{-6}$ bar, creating melilite + spinel + clinopyroxene, the characteristic mineral assemblage of Type B CAIs. For $1 \times 10^{-4} \geq P^{\text {tot }} \geq 2 \times 10^{-6}$ bar, this causes the $\mathrm{SiO}_{2}$ content to rise to $>35 \mathrm{wt} \%$ where the melilite is exhausted before forsterite condenses. At $P^{\text {tot }} \geq 1 \times 10^{-3}$ bar, however, forsterite condenses before melilite is exhausted and the condensation path follows a relatively low- $\mathrm{SiO}_{2}$ trajectory to the right on Figure $4 \mathrm{~b}$. Within this range of $P^{\text {tot }}$, the melilite + spinel + clinopyroxene segment shifts to progressively lower $\mathrm{MgO}$ contents with decreasing $P^{\text {tot }}$. No silicate liquids are calculated to be stable at $P^{\text {tot }} \leq 10^{-1}$ bar for a system of solar composition.

At lower $P^{t o t},<2 \times 10^{-6}$ bar, melilite begins to react to form anorthite before much clinopyroxene forms, and the condensation paths veer toward the upper left on Figure 4a. A continuous series of condensation paths, corresponding to a relatively narrow interval of $P^{t o t}$, sweeps through the very large region of $\mathrm{SiO}_{2}-\mathrm{MgO}$ space that lies between the paths shown for $1 \times 10^{-7}$ and $1.2 \times 10^{-6}$ bar. It should be noted that chemical equilibrium requires that the large number of gas-solid reactions implied by the multiple segments between points $\mathrm{A}$ and $\mathrm{B}$ along the path for $1 \times 10^{-7}$ bar in Figure $4 \mathrm{a}$ all occur within a temperature interval of only $12 \mathrm{~K}$. This temperature interval and the number of phase appearances and disappearances shrink as $P^{\text {tot }}$ increases from $1 \times 10^{-7}$ to $1.2 \times 10^{-6}$ bar. No anorthite stability field was found at $2 \times 10^{-6}$ bar but, at $1 \times 10^{-5}$ bar (not shown), anorthite forms more than $50 \mathrm{~K}$ below the forsterite condensation temperature, by reaction of spinel with clinopyroxene. This indicates that the much-discussed crossover $P^{\text {tot }}$ above which forsterite condenses at a higher temperature than anorthite is $\sim 2 \times 10^{-6}$ bar, rather than $\sim 2.5 \times 10^{-8}$ (Petaev and Wood, 1998), $\sim 3.8 \times 10^{-4}$ (MacPherson et al., 2004), or $\sim 10^{-5}$ bar (MacPherson et al., 2005). The reasons for the differences among the latter three estimates and between them and the value obtained here are unknown, as neither the elemental abundances, nor the thermodynamic data sources nor the nature of the clinopyroxene solution model are given in the latter three papers.

\subsection{Condensation paths for dust-enriched systems}

Fedkin and Grossman (2006) pointed out that the oxidation state of iron in chondrites indicates that much of the material in them formed in systems whose oxygen fugacity, $f_{\mathrm{O}_{2}}$, was much higher than that expected in a system of solar composition. It has been known since the work of Wood (1967) that one way of enhancing the $f_{\mathrm{O}_{2}}$ of a cosmic gas is by total evaporation of a system which had become enriched in dust relative to gas but is otherwise solar in composition. Ebel and Grossman (2000) showed

that not only does the $f_{\mathrm{O}_{2}}$ differ from that of a system of solar composition during condensation in such systems, but so also do condensation sequences and, hence, 
condensation paths. If CAIs condensed from dust-enriched systems, then their compositions should be compared to condensation paths relevant to such conditions, and those paths may differ from those in Figures $4 \mathrm{a}$ and $4 \mathrm{~b}$. The atomic $T i^{3+} /\left(T i^{3+}+T i^{4+}\right)$ ratios of fassaite in CAIs are sensitive to $f_{\mathrm{O}_{2}}$, and range from $\sim 0.3$ to $\sim 0.8$, with a mean of $\sim 0.54$. Grossman et al. (2007a) showed that such values indicate that CAIs formed at an $f_{\mathrm{O}_{2}}$ very close to that of a system of solar composition at $1500 \mathrm{~K}$. For OC, the anhydrous chondritic dust component defined by Yoneda and Grossman (1995), enrichment in dust relative to gas by factors of 10 and 100 compared to solar composition results in enhancements in $f_{\mathrm{O}_{2}}$ by factors of 8 and 120 , respectively, at $1500 \mathrm{~K}$. Using $-\ln f_{O_{2}} \propto 4 \ln X_{T i^{3+}} / X_{T_{i} i^{4+}}$, it can be shown that, if the $f_{O_{2}}$ of CAI formation were 8 times higher than the solar value, the range of $T i^{3+} /\left(T i^{3+}+T i^{4+}\right)$ ratios in fassaite would be little different from the observed range but, if the $f_{O_{2}}$ were 120 times higher than the solar value, the range of $T i^{3+} /\left(T i^{3+}+T i^{4+}\right)$ ratios would be from $\sim 0.1$ to $\sim 0.5$, significantly different from the observed distribution. Accordingly, to model condensation at a dust enrichment plausibly relevant to CAI formation, a set of condensation calculations identical to that described above was carried out for a system enriched in OC dust by a factor of 10 .

The resulting condensation paths are shown in Figures $4 \mathrm{c}$ and $4 \mathrm{~d}$. Because the condensation sequences are so similar, the dust enrichment paths for $10^{-7} \leq P^{\text {tot }} \leq 2 \times 10^{-5}$ bar are very similar to the solar composition paths for $2 \times 10^{-6} \leq P^{\text {tot }} \leq 1 \times 10^{-3}$ bar, and the dust enrichment paths for $5 \times 10^{-5} \leq P^{t o t} \leq 1 \times 10^{-3}$ bar are very similar to the solar composition paths for $3 \times 10^{-3} \leq P^{\text {tot }} \leq 1 \times 10^{-1}$ bar. Furthermore, as in solar composition, forsterite condensation prior to much clinopyroxene formation leads to a family of condensation paths extending to high $\mathrm{MgO}$ contents at intermediate $\mathrm{SiO}_{2}$ contents over a narrow range of $P^{\text {tot }}$, between $2 \times 10^{-5}$ and $5 \times 10^{-5}$ bar in this case. For $4 \times 10^{-3} \leq P^{t o t} \leq 1 \times 10^{-1}$ bar, however, the dust enrichment paths are distinct from any of the solar composition paths seen in Figures $4 \mathrm{a}$ and $4 \mathrm{~b}$. This is due to the stability of CMAS liquids at these pressures for the dust enrichment considered here. Within this range of $P^{t o t}$, the liquid stability field increases in size with increasing $P^{\text {tot }}$. On the path for $1 \times 10^{-2}$ bar, liquid forms along the near-vertical segment at $\sim 10 \mathrm{wt} \% \mathrm{MgO}$, and results from the reaction of melilite with the gas to form a CMAS condensate liquid with falling temperature. That path continues as a straight line proceeding toward the upper right with falling temperature, along which spinel + liquid \pm perovskite is the stable phase assemblage before being joined by forsterite. At $10^{-1}$ bar, in contrast, CMAS liquid + spinel \pm perovskite is the stable phase assemblage even for $\mathrm{MgO}$ contents below $10 \mathrm{wt} \%$, replacing the high-temperature melilite stability field found at $1 \times 10^{-2}$ bar. At intermediate $P^{t o t}, 1.1 \times 10^{-2}$ bar, the stability field of CMAS liquid + spinel \pm perovskite is split, with melilite crystallizing from the liquid with falling temperature and then reacting with the gas at still lower temperature to form CMAS liquid again. Because the melilite-liquid phase relations are very poorly predicted by the CMAS liquid model employed here (Grossman et al., 2002), the paths 
shown at the extreme left of Figures $4 \mathrm{c}$ and $4 \mathrm{~d}$ should be considered quite uncertain. This is of little consequence to the conclusions of this work.

\subsection{Bulk compositions of CAIs compared to condensation paths}

For each inclusion, a point corresponding to the $\mathrm{SiO}_{2}$ and $\mathrm{MgO}$ contents given in Table 4 is plotted as a bold symbol in each of Figures $5 \mathrm{a}$ and $5 \mathrm{~b}$, filled for Type A inclusions and open for Type Bs. Condensation paths for solar composition are shown for reference, at relatively low values of $P^{\text {tot }}$ in Figure $5 \mathrm{a}$ and relatively high $P^{\text {tot }}$ in Figure 5b. The plain symbols that surround the bold symbol of the same shape correspond to the compositions generated by the possible variations in mineral proportions that were averaged to yield the composition indicated by the bold symbol. In each of Figures $5 \mathrm{a}$ and $5 \mathrm{~b}$, each inclusion thus plots as a field, circumscribed by a solid curve if it is a Type A and a dotted curve if a Type B, within which lies the actual composition. The reason why these fields are not described by the usual orthogonal error bars is that they do not arise from uncertainty in measurements of independent parameters but rather from correction for assumed over- or under-sampling of stoichiometric phases with specific $\mathrm{Mg} / \mathrm{Si}$ ratios.

Inspection of Figures 5a and 5b reveals that Type B inclusions, excluding E60, have higher average $\mathrm{SiO}_{2}$ and $\mathrm{MgO}$ contents, 27.6 \pm 2.1 and $11.7 \pm 1.0 \mathrm{wt} \%$, respectively, than Type As, 22.1 \pm 2.6 and 9.4 $\pm 2.0 \mathrm{wt} \%$. Furthermore, while almost all Type Bs have higher $\mathrm{SiO}_{2}$ contents than almost all Type As, there is more overlap in the $\mathrm{MgO}$ contents of the two inclusion types. The classification of inclusion E107 is problematic. While it contains only $\sim 20 \mathrm{vol} \%$ fassaite, which would make it a Type A, it also contains a few grains of primary anorthite, which would make it a Type B. Furthermore, its fassaite contains an average of $\sim 9 \mathrm{wt} \%$ total Ti oxides, which is less than the minimum level seen in fassaite in CTAs, $\sim 15 \mathrm{wt} \%$ (Simon et al., 1999). E107 is thus classified a Type B in this work. Appropriately, in Figures 5a and 5b, its bulk composition plots in the border region between the Type A and Type B fields.

Inspection of Figures $5 \mathrm{a}$ and $5 \mathrm{~b}$ shows that the observed compositions of six of the eight Type A inclusions lie within error of the calculated condensation path for $1.2 \times 10^{-6}$ bar, while that of E49A is compatible with the path for $3 \times 10^{-3}$ bar and that for E13 would lie on one of the family of paths lying between $1.2 \times 10^{-6}$ and $1 \times 10^{-7}$ bar. In contrast, five of the nine Type Bs would lie within that family of paths, three others on the curves that would lie between those for $1.2 \times 10^{-6}$ and $1 \times 10^{-4}$ bar, and the final one, E60, on a path near that for $1 \times 10^{-3}$ bar. Compared to the condensation paths for $1 \times 10^{-}$ ${ }^{4} \leq P^{\text {tot }} \leq 1 \times 10^{-1}$ bar, the observed compositions of all Type $\mathrm{B}$ inclusions are too low in $\mathrm{MgO}$ for their $\mathrm{SiO}_{2}$ contents, just as was noted by Grossman et al. (2000).

The same data plotted in Figures $5 \mathrm{a}$ and $5 \mathrm{~b}$ are plotted in Figures $6 \mathrm{a}$ and $6 \mathrm{~b}$, where they are compared to condensation paths for systems enriched in OC dust by a 
factor of ten. The dust enrichment path for $10^{-7}$ bar passes through the fields of five of the eight Type A inclusions and very close to that for a sixth, E55, while the compositions of E13 and E49A would require $P^{t o t} \geq 10^{-2}$ bar and $\sim 10^{-4}$ bar, respectively. While the compositions of three of the nine Type B CAIs lie along the path for $10^{-2}$ bar and that of TS33 lies very close to it, those of E107 and TS34 would lie close to a curve in the vicinity of $4-6 \times 10^{-3}$ bar, that of E60 lies on the path for $2 \times 10^{-5}$ bar, and that of 1623-8 would lie close to a curve in the vicinity of $1 \times 10^{-6}$ bar, no dust enrichment path has been found that is compatible with the composition of F7.

Grossman et al. (2000) assumed that the heavy isotope enrichments of $\mathrm{Mg}$ and $\mathrm{Si}$ in CAIs are the result of partial evaporation of these elements after the inclusions condensed, and argued that the present-day compositions of the inclusions, such as those plotted in Figs. 5 and 6, were modified in that evaporation event. In the following sections, the $\mathrm{Mg}$ and $\mathrm{Si}$ isotopic compositions are used to estimate the bulk chemical compositions of the inclusions prior to evaporation in order to see if their unmodified compositions agree with predictions of condensation models.

\subsection{Fractions of Mg and Si evaporated}

Due to the possible presence of excess ${ }^{26} \mathrm{Mg}$ from decay of extinct ${ }^{26} \mathrm{Al}$ in these samples, determination of $\mathrm{F}_{\mathrm{Mg}}$, the $\mathrm{Mg}$ mass fractionation, is based on the $\delta^{25} \mathrm{Mg}$ data. For each inclusion for which multiple measurements of $\delta^{25} \mathrm{Mg}$ were made, a mean value was obtained by weighting the individual measurements on Table 2 by the inverse square of their uncertainties. These data are shown in Table 4, along with literature values for three other inclusions, F11, F7 and 1623-8. Because the terrestrial Mg standard employed in this work has the same isotopic composition as Orgueil (Galy et al., 2003), it is assumed that the $\mathrm{F}_{\mathrm{Mg}}$ data for the inclusions are relative to the mean $\mathrm{Mg}$ isotopic composition of condensed solar system matter.

For each inclusion, the mean $\delta^{30} \mathrm{Si}$ on Table 2 was divided by 2 to obtain $\mathrm{F}_{\mathrm{Si}}$, the Si mass fractionation. Molini-Velsko et al. (1986) found that bulk meteorites have $\delta^{30} \mathrm{Si}$ of $-0.55 \%$ relative to NBS-28, indistinguishable from the value of $-0.58 \pm 0.06 \%$ ound by Georg et al. (2007), so the former was assumed to represent average solar system matter and the isotopic composition of CAIs when they condensed. Since all silicon isotopic data on Table 2 and those taken from the literature for TS34, F11, F7 and 1623-8

were measured relative to NBS-28, all the $\mathrm{F}_{\mathrm{Si}}$ data used in this work were increased by $0.275 \%$ in order to express their deviation from mean solar system matter. These are the data given in Table 4.

For those samples whose isotopic compositions were measured in this work, the $\mathrm{F}_{\mathrm{Si}}$ and $\mathrm{F}_{\mathrm{Mg}}$ data on Table 4 are plotted against one another in Figure $7 \mathrm{a}$ for Type A inclusions and in Figure $7 \mathrm{~b}$ for Type Bs. All of the data from the study of Grossman et al. (2000) are also plotted on Figures $7 \mathrm{a}$ and $7 \mathrm{~b}$, and these include the literature data for 
F11, F7 and 1623-8 that also appear on Table 4. Among the four Type A inclusions whose isotopic data were summarized by Grossman et al. (2000), there is a cluster of three having $\mathrm{F}_{\mathrm{Si}}$ between 1 and $2.6 \%$ /amu and $\mathrm{F}_{\mathrm{Mg}}$ between 5 and $11 \%$ /amu, and one has negative $\mathrm{F}_{\mathrm{Si}}$ with relatively low $\mathrm{F}_{\mathrm{Mg}}$ of $\sim 2 \%$ /amu. Among the seven Type As analysed in this work, there is a similar cluster of five with $\mathrm{F}_{\mathrm{Si}}$ between 1 and $2.2 \%$ /amu but their $\mathrm{F}_{\mathrm{Mg}}$ are 1-8 \%o/amu, in a slightly lower range than their counterparts in the earlier work. The other two Type As studied here, E13 and E62, have negative $\mathrm{F}_{\mathrm{Mg}}$, and one of the latter, E13, also has negative $\mathrm{F}_{\mathrm{Si}}$. Among the Type Bs considered by Grossman et al. (2000), $\mathrm{F}_{\mathrm{Si}}$ is positively correlated with $\mathrm{F}_{\mathrm{Mg}}$, and this is also the case for the Type Bs analysed in the present study, the major difference being that the most fractionated inclusion in the present work has $F_{S i}$ and $F_{M g}$ of 2.1 and $6.1 \%$ /amu, respectively, much less than the most fractionated inclusion in the earlier work whose $\mathrm{F}_{\mathrm{Si}}$ and $F_{\mathrm{Mg}}$ are 3.8 and $9.0 \%$ /amu. None of the Type Bs in either study has negative $\mathrm{F}_{\mathrm{Si}}$ or $\mathrm{F}_{\mathrm{Mg}}$.

If, as in Grossman et al. (2000), it is assumed that the heavy isotope enrichments in $\mathrm{Mg}$ and $\mathrm{Si}$ result from kinetic isotope fractionation that occurred during evaporation into a low-density gas while the inclusions were molten, the fraction of each element remaining, $f_{r e m}$, can be calculated using the Rayleigh equation,

$$
R / R_{o}=f_{r e m}^{\alpha-1}
$$

where $\alpha$ is the isotopic fractionation factor for evaporation of the element in question from this specific matrix, $R$ is the observed isotope ratio of the element and $R_{O}$ is the initial isotope ratio, here taken as the mean isotopic composition of the element in condensed solar system matter.

Isotopic fractionation factors have been determined for $\mathrm{Mg}$ and $\mathrm{Si}$ by measuring the isotopic compositions of residues produced by evaporation of CMAS liquid compositions similar to those of Type B inclusions in vacuum and hydrogen as a function of temperature. Knight et al. (2007) found that (1- $\alpha$ ) for $\mathrm{Si}$ evaporation is $0.0103 \pm 0.0007$, independent of temperature. Richter et al. (2005) found that (1- $\alpha$ ) for $\mathrm{Mg}$ evaporation is a linear function of inverse temperature. Using petrologic constraints, Grossman et al. (2002) suggested that the average temperature for evaporation of Type A CAI liquid compositions is $\sim 1775 \mathrm{~K}$ and that for Type Bs is $\sim 1675 \mathrm{~K}$. At $1775 \mathrm{~K},(1-\alpha)$ for $\mathrm{Mg}$ is $0.01203 \pm 0.00073$ and, at $1675 \mathrm{~K}$, the extrapolated value is 0.011 .

For each inclusion in Table 4, the fraction of each of the $\mathrm{Mg}$ and $\mathrm{Si}$ evaporated was calculated from the isotopic composition for each element and the element's fractionation factor at the appropriate temperature for each inclusion type. Excluded from this calculation are the Type A inclusions, E13 and E62, because of their negative $\mathrm{F}_{\mathrm{Si}}$ and/or $\mathrm{F}_{\mathrm{Mg}}$. The significance of these light isotope enrichments will be discussed later. Results for the remaining inclusions are plotted in Figure 8. There appears to be 
little difference in the degree of evaporation of these elements between Types A and B inclusions.

\subsection{Initial chemical compositions of CAIs}

For each inclusion plotted in Figure 8, the fractions of $\mathrm{Mg}$ and $\mathrm{Si}$ evaporated were combined with the present-day bulk chemical composition in Table 4 to obtain the initial bulk chemical composition, which is listed in Table 5. These are the compositions that each CAI had before evaporation caused $\mathrm{Mg}$ and $\mathrm{Si}$ loss, referred to herein as the preevaporation compositions. The pre-evaporation $\mathrm{SiO}_{2}$ and $\mathrm{MgO}$ contents are plotted in Figures $9 \mathrm{a}$ and $9 \mathrm{~b}$, where they are compared to condensation paths for solar composition, and in Figures 10a and 10b, where they are compared to condensation paths for dust enrichment. For each inclusion, every member of the cluster of points representing the possible range of present-day compositions in Figures 5 and 6, is itself transformed into a new cluster of points in Figures 9 and 10 due to propagation of the uncertainty in $\mathrm{Mg}$ and $\mathrm{Si}$ isotopic compositions. For clarity, the latter uncertainty is not propagated for the mean composition of each inclusion, which is again plotted as a bold symbol. For each inclusion, the pre-evaporation composition lies within the field that circumscribes the plain symbols for that inclusion. Inspection of Figures 9 and 10 reveals that the preevaporation compositions of Type Bs are even more clearly separated from those of Type As than are the observed compositions in Figures 5 and 6 . While there is still considerable overlap in $\mathrm{MgO}$ content between the two inclusion types, the average preevaporation $\mathrm{SiO}_{2}$ content of Type Bs, excluding the forsterite-bearing one, E60, is $28.9 \pm 2.6 \mathrm{wt} \%$, while that of Type As is only $23.4 \pm 2.6 \mathrm{wt} \%$. Inclusion E107 again has a composition falling between most Type A and most Type B inclusions.

In comparing chemical compositions of CAIs with condensation paths for solar composition, it is clear that the pre-evaporation compositions in Figures $9 \mathrm{a}$ and $9 \mathrm{~b}$ are shifted toward condensation paths for higher $P^{\text {tot }}$ compared to their present-day compositions in Figures 5a and 5b, respectively. It is also clear that no single condensation path can pass through all inclusion compositions. The composition fields of a remarkably large fraction of the inclusions, however, four of six Type As and six of nine Type Bs, are intersected by a subset of the condensation paths calculated for a relatively narrow range of $P^{\text {tot }}$, between $1.2 \times 10^{-6}$ and $1 \times 10^{-4}$ bar. The Type A exceptions are E56, which would seem to require $P^{\text {tot }}$ below $1.2 \times 10^{-6}$ bar, and TS32, requiring $P^{\text {tot }}$ slightly greater than $10^{-1}$ bar. The Type B exceptions are TS34 and 1623-8, requiring $P^{t o t}$ of $\sim 2 \times 10^{-3}$ bar and slightly greater than $10^{-1}$ bar, respectively, and the forsteritebearing inclusion, E60, requiring $P^{\text {tot }}$ of $\sim 10^{-3}$ bar. Recall, however, that 1623-8 and E60 are two of the CAIs whose compositions were determined by INAA, so it is conceivable that their actual compositions lie slightly outside their fields on Figure 9. Similarly, when pre-evaporation compositions are compared with condensation paths for 
enrichment in OC dust by a factor of ten in Figures 10a and 10b, it is again clear that no single path can account for all inclusion compositions. The path for $P^{t o t}=1.1 \times 10^{-2}$ bar passes through the fields of five of the nine Type Bs and one of the six Type As, and comes very close to that for another Type A, E56. Another path, at $P^{\text {tot }}=3 \times 10^{-5} \mathrm{bar}$, would pass through the fields of three of the remaining Type As and one of the remaining Type Bs. This leaves only four inclusions unaccounted for. E107 and 1623-8, Type Bs, require $P^{\text {tot }}$ near $1 \times 10^{-7}$ and slightly greater than $1 \times 10^{-3}$ bar, respectively. TS32, a Type A, also requires $P^{\text {tot }}$ slightly higher than $1 \times 10^{-3}$ bar, and E60 requires $P^{\text {tot }}$ of $\sim 5 \times 10^{-2}$ bar.

In summary, compatibility of most pre-evaporation compositions with condensation paths for dust enrichment tends to occur at higher values of $P^{\text {tot }}$ than with those for solar composition. Regardless of which set of condensation paths is used for comparison, the variation in pre-evaporation compositions requires that the population of inclusions studied here originated in nebular zones of widely different $P^{\text {tot }}$. Ruden and Pollack (1991) calculated the variation of the midplane temperature and pressure with time and distance from the center of the solar nebula for three different disk masses and a single turbulence parameter. In all cases considered, wherever and whenever the temperature is within the range of silicate condensation temperatures, $2000-1000 \mathrm{~K}, P^{\text {tot }}$ lies between $6 \times 10^{-6}$ and $6 \times 10^{-3}$ bar, a range little different from that with which most of the above inclusions are compatible.

Much of the variation in $P^{\text {tot }}$ inferred from Figures 9 and 10 is due to a relatively wide range of $\mathrm{MgO}$ in the pre-evaporation compositions. In both figures, a significant number of inclusions plot within a family of condensation paths, representing a range in $P^{\text {tot }}$, along which a sharp rise in $\mathrm{SiO}_{2}$ content occurs from $\sim 21 \mathrm{wt} \%$ to $\sim 36 \mathrm{wt} \%$. The increase in $\mathrm{SiO}_{2}$ content is due to reaction of akermanitic melilite with the gas to form diopsidic clinopyroxene. With increasing $P^{\text {tot }}$, this increase occurs at higher $\mathrm{MgO}$ content, due to higher åkermanite content of melilite, beginning at $\sim 11 \mathrm{wt} \%$ at $2 \times 10^{-6}$ bar on Fig. 9a and $\sim 14 \mathrm{wt} \%$ at $1.3 \times 10^{-3}$ bar on Fig. 9b, for example. If the increase in the åkermanite content of melilite, a diffusion-controlled process, had failed to keep up with falling temperature at any particular $P^{\text {tot }}$, clinopyroxene condensation might have begun when the bulk assemblage had a lower $\mathrm{MgO}$ content than predicted by the model. A wide range of bulk $\mathrm{MgO}$ contents could conceivably result from condensation at fixed $P^{t o t}$ if different inclusions were affected differentially by this departure from equilibrium.

Pre-evaporation compositions are expected to fall along calculated condensation paths only if each inclusion is a representative sample of the non-metallic condensates that existed at a given temperature in a system of the assumed composition and pressure. At temperatures below that where melilite reacts to form clinopyroxene, forsteritic olivine is the next major condensate mineral to form along most condensation paths shown in Figs. 9 and 10. Forsterite differs from spinel, melilite and clinopyroxene in that its condensation does not involve reaction of pre-existing solid phases with the gas. Thus, all of the major components of forsterite condense directly from the gas. In order 
for a preexisting nodule of spinel + clinopyroxene to remain on a given condensation path after forsterite formation, the nodule must mix with independently condensed forsterite in the exact proportions in which they occur in the nebula. Because the condensate forsterite plots at $\sim 43 \mathrm{wt} \% \mathrm{SiO}_{2}$ and $\sim 56 \mathrm{wt} \% \mathrm{MgO}$, addition of slightly more or less than the equilibrium proportions of this phase at a given $P^{\text {tot }}$ could broaden the distribution of pre-evaporation compositions in Figs. 9 and 10 mostly in the $\mathrm{MgO}$ direction, making them appear to represent condensates from a wide range of $P^{\text {tot }}$.

\subsection{Forward kinetic modeling of evaporative change in composition}

In this work, the original chemical compositions of CAIs were calculated from their observed compositions using isotopic data only, under the assumption that the heavy isotope enrichments of $\mathrm{Mg}$ and $\mathrm{Si}$ are the result of evaporation of partially molten CAIs. As a test of this assumption, forward kinetic modeling was performed to see if each observed chemical composition could be regenerated from its corresponding preevaporation composition using only thermodynamic and kinetic data for CMAS liquids, completely independent of the isotopic data. In performing this exercise, it is not sufficient merely to regenerate any composition within the field for a CAI in Figure 5 from a starting point that lies anywhere within its respective field in Figure 9. This is because the isotopic compositions were used to translate each point that defines the field for a given CAI in Figure 5, into a corresponding point in Figure 9 that was broadened into a cluster of points by propagating the uncertainty in the isotopic compositions. The area of that cluster of points is only a fraction of the area occupied by the field for a given CAI in Figure 9. The exercise can only be considered a success if any point within a small cluster of points in Figure 9 can be used to regenerate its point of origin in Figure 5.

Kinetic modeling of the change in composition with progressive evaporation was performed as in Grossman and Fedkin (2003) but with some significant differences. A droplet radius of $0.25 \mathrm{~cm}$, an ambient $P_{H}^{\text {tot }}$ of $1 \times 10^{-3}$ bar, and a closed system of solar composition were assumed. The assumption that the $\mathrm{Ca} / \mathrm{Al}$ ratio is solar in each preevaporation composition implies that $100 \%$ of the $\mathrm{Al}$ and $\mathrm{Ca}$ were condensed into the droplet. The initial distributions of $\mathrm{Mg}$ and $\mathrm{Si}$ between droplet and gas were calculated by comparing the $\mathrm{Mg} / \mathrm{Al}$ and $\mathrm{Si} / \mathrm{Al}$ ratios of the pre-evaporation composition to their respective solar ratios. In addition to these four elements, the system was assumed to contain $\mathrm{H}, \mathrm{He}, \mathrm{C}, \mathrm{N}, \mathrm{O}, \mathrm{P}$ and $\mathrm{S}$ in solar proportions. At each evaporation step, gas-phase equilibrium was used to compute the partial pressures, $P_{i}^{a}$, of all significant species i in the ambient gas, uninfluenced by the droplet. The vapor pressures, $P_{i}^{v}$, of $\mathrm{Mg}_{(\mathrm{g})}$ and $\mathrm{SiO}_{(\mathrm{g})}$ were calculated at each step from the equilibria,

$$
M g O_{(l)} \Leftrightarrow M g_{(g)}+1 / 2 O_{2(g)}
$$


and

$$
\mathrm{SiO}_{2(l)} \Leftrightarrow \mathrm{SiO}_{(g)}+1 / 2 \mathrm{O}_{2(g)}
$$

using the Berman (1983) relationship between component activities and composition in CMAS liquids, and the $f_{\mathrm{O}_{2}}$ of the ambient gas. This is equivalent to assuming that the droplet and ambient gas were in redox equilibrium during evaporation, an assumption that is justified by the fact that the oxidation state of $\mathrm{Ti}$ in real CAIs is that expected from equilibrium in a system of solar composition. The flux of each species, $J_{i}$, is calculated from the Hertz-Knudsen equation which, for $\mathrm{Mg}_{(\mathrm{g})}$, for example, is written

$$
J_{M g}=\frac{\alpha_{M g}\left(P_{M g}^{v}-P_{M g}^{a}\right)}{\sqrt{2 \pi M R T}}
$$

where $\alpha_{M g}, \mathrm{M}, \mathrm{R}$ and $\mathrm{T}$ are the evaporation coefficient and molecular weight of $\mathrm{Mg}_{(\mathrm{v})}$, the gas constant and temperature, respectively. Data for $\alpha_{M g}$ and its temperature dependence were taken from evaporation experiments on CMAS liquids by Richter et al. (2002), and $\alpha_{S i O}$ was assumed to be $\alpha_{M g} \times 1.425$, as found at $1500 \mathrm{~K}$ in $\mathrm{H}_{2}$ in the same work. The composition change in each evaporation step was obtained by multiplying the flux of each species by the time step size and the surface area of the droplet, the latter reduced by the computed equilibrium volume proportions of crystalline spinel and melilite.

The strategy was to use the pre-evaporation composition as the starting material, and to calculate the change in its composition due to isothermal evaporation in order to see how close the evolutionary path comes to the specific observed composition from which the pre-evaporation composition was inferred. Various pairs of pre-evaporation and observed compositions were attempted for each inclusion. Because experimental petrologic evidence reviewed by Grossman et al. (2002) suggests that peak temperatures experienced by compact Type As and Type Bs were $\sim 1773$ and $\sim 1673 \mathrm{~K}$, respectively, evaporation temperatures selected for modeling were usually within $\pm 50 \mathrm{~K}$ of these values.

Shown in Figure 11 for each inclusion is the pair of pre-evaporation and observed compositions for which the computed evaporation curve comes closest to the observed composition. For the assumptions stated above, results are very good to excellent for four of six Type A inclusions. Results for the remaining two Type As, E49A and E55, are very poor. The rate of evaporation of $\mathrm{Mg}$ relative to that of $\mathrm{Si}$ increases with the $\mathrm{Mg} / \mathrm{Si}$ ratio of the starting material, and this effect is clearly seen in comparing the slopes of the model curves for E49 and E55, which were computed at the same temperature. Although the pre-evaporation compositions of E49A and E49 selected for Fig. 11 are almost identical, their corresponding observed compositions are quite different, implying that their thermal histories are different. Thus, while the observed composition of E49 is 
intersected perfectly by the isothermal evaporation curve for $1723 \mathrm{~K}$, the lower limit temperature used for modeling Type As, the observed composition of E49A is poorly fit even when the model temperature is $50 \mathrm{~K}$ lower, as illustrated in Fig. 11. For E55, there is no temperature for which an evaporation curve can be found that joins the preevaporation and observed compositions. It is worth noting, however, that, E49A and E55 are the only Type As plotted whose observed compositions were determined by INAA, so the uncertainties on their compositions may be greater than estimated, for the reasons stated above.

Results for Type Bs are excellent except for E60, for which the curve misses by $1 \% \mathrm{SiO}_{2}$. There are two possible reasons why the modeling result for this inclusion is poorer than for the other Type Bs. First, this is another object for which the observed composition was obtained by INAA. Second, it has an unusual, Mg-rich starting composition whose mineral assemblage was undoubtedly forsterite-rich. There are no existing constraints on evaporation temperatures of such objects, so the selected model temperature, though the same as those used for other Type Bs, may not be appropriate for this composition.

\subsection{Refractory element enrichment factors}

For each sample in Figure 8, the $\mathrm{wt} \% \mathrm{CaO}+\mathrm{Al}_{2} \mathrm{O}_{3}$ is plotted against the wt $\%$ $\mathrm{MgO}+\mathrm{SiO}_{2}$ for the observed compositions (Table 4) in Figure 12a and for the preevaporation compositions (Table 5) in Figure 12b. Types A and B inclusions are plotted as filled and open symbols, respectively. The inverse linear correlations are a simple consequence of the fact that these are the only four oxide components considered here. Shown for reference are a series of lines radiating from the origin whose slopes are equal to the specified multiples of the solar system $\left(\mathrm{CaO}+\mathrm{Al}_{2} \mathrm{O}_{3}\right) /\left(\mathrm{MgO}+\mathrm{SiO}_{2}\right)$ ratio of 0.07502. The average observed Type $\mathrm{A}$ and Type $\mathrm{B}$ compositions are plotted on Figure $12 \mathrm{a}$, and the average pre-evaporation compositions on Figure $12 \mathrm{~b}$.

Among the observed compositions, Type As are enriched in $\left(\mathrm{CaO}+\mathrm{Al}_{2} \mathrm{O}_{3}\right) /(\mathrm{MgO}$ $+\mathrm{SiO}_{2}$ ) relative to the solar system abundances by factors of 24 to 33; Type Bs by factors of 17 to 24. This is merely a reflection of the higher $\mathrm{MgO}$ and $\mathrm{SiO}_{2}$ contents of Type $\mathrm{Bs}$ that was previously noted. For Type As, the average enrichment factor of $(\mathrm{CaO}+$ $\left.\mathrm{Al}_{2} \mathrm{O}_{3}\right) /\left(\mathrm{MgO}+\mathrm{SiO}_{2}\right)$ is 29.0 relative to the solar system abundances; for Type $\mathrm{Bs}$, it is 20.8 .

Among the pre-evaporation compositions, Type As are enriched in $(\mathrm{CaO}+$ $\left.\mathrm{Al}_{2} \mathrm{O}_{3}\right) /\left(\mathrm{MgO}+\mathrm{SiO}_{2}\right)$ relative to the solar system abundances by factors of 21 to 29 ; Type Bs by factors of 13 to 21 . For Type As, the average enrichment factor of $(\mathrm{CaO}+$ $\left.\mathrm{Al}_{2} \mathrm{O}_{3}\right) /\left(\mathrm{MgO}+\mathrm{SiO}_{2}\right)$ is 23.3 relative to the solar system abundances; for Type $\mathrm{Bs}$, it is 16.4. The lower enrichments in relatively refractory $\mathrm{CaO}+\mathrm{Al}_{2} \mathrm{O}_{3}$ compared to relatively volatile $\mathrm{MgO}+\mathrm{SiO}_{2}$ in the pre-evaporation compositions compared to the observed 
compositions is due to removal of the effects of evaporation from the pre-evaporation compositions. Dividing the average enrichment factors for the pre-evaporation compositions by the averages for the observed compositions yields the fraction of the refractory element enrichment due to initial condensation. For Type As, $80.3 \%$ of the refractory element enrichment is due to initial condensation and $19.7 \%$ due to subsequent evaporation. For Type Bs, the corresponding proportions are $78.9 \%$ and $21.15 \%$.

\subsection{Implications of inclusions with enrichments in light isotopes}

The negative $\mathrm{F}_{\mathrm{Si}}$ and/or $\mathrm{F}_{\mathrm{Mg}}$ of the two Type A inclusions, E13 and E62, in Table 4 precludes computation of their pre-evaporation compositions. Such light-isotope enrichments may be due to a kinetic isotope effect accompanying condensation. Why these two objects are the only ones in this study that show this feature is unknown. The size of such an effect may, however, be related to the degree of isotope exchange or backreaction during evaporation in a closed system. Grossman and Fedkin (2003) presented a model study of chemical and isotopic effects of evaporation of Mg and Si from CMAS liquids into a closed system of solar composition. In cases where evaporation occurred during cooling of the droplet, they found a narrow temperature/time interval for each element within which the flux reversed direction, and evaporation was replaced by condensation with falling temperature. In such circumstances, isotopic exchange led to very large reductions in $\delta^{25} \mathrm{Mg}$ of the droplet just before and just after net condensation of $\mathrm{Mg}$ began, sometimes even resulting in negative $\delta^{25} \mathrm{Mg}$, before a significant increase in the $\mathrm{MgO}$ content of the droplet occurred. While the flux reversal occurred at very low $\mathrm{Mg}$ concentration in the droplet, an analogous effect might happen at higher $\mathrm{Mg}$ content if evaporation occurs in a dust-enriched system. Upon discovering several coarse-grained inclusions whose $\mathrm{Ca}$ was mass-fractionated in favor of light isotopes and whose $\mathrm{Mg}$ was mass-fractionated in favor of heavy isotopes, Niederer and Papanastassiou (1984) suggested that the $\mathrm{Ca}$ isotopic composition reflected formation of early condensates while the $\mathrm{Mg}$ isotopic composition recorded an evaporation process.

If a kinetic isotope effect during condensation or back-reaction is the cause of the negative mass-fractionations in E13 and E62, it may have also affected the isotopic compositions of the other CAIs without reducing their $\mathrm{F}_{\mathrm{Si}}$ or $\mathrm{F}_{\mathrm{Mg}}$ to negative values. If so, the strategy adopted herein of using the Rayleigh equation to calculate preevaporation chemical compositions of inclusions from their $\mathrm{F}_{\mathrm{Si}}$ and $\mathrm{F}_{\mathrm{Mg}}$ data would be seriously flawed. If, however, such isotopic exchange had significantly affected the CAIs studied here, it is likely that much greater difficulty would have been encountered in recovering their observed compositions by forward kinetic modeling of pre-evaporation compositions inferred from the Rayleigh equation. Perhaps this is the reason for the relatively poor agreement between model evaporation curves and the observed compositions of E55, E49A and E60. 


\section{CONCLUSIONS}

When the bulk chemical compositions of samples of Types A and B coarsegrained inclusions from $\mathrm{CV} 3$ chondrites are corrected for non-representative sampling in the laboratory and then corrected for evaporative losses inferred from their $\mathrm{Mg}$ and $\mathrm{Si}$ isotopic compositions assuming Rayleigh fractionation, the resulting pre-evaporation chemical compositions are consistent with those predicted for high-temperature condensates from equilibrium thermodynamic calculations. To explain all preevaporation compositions in this way, however, different inclusions must have condensed from nebular regions differing in total pressure by a factor of $10^{5}$, regardless of whether they formed in a system of solar composition or in one enriched in OC dust relative to gas by a factor of ten relative to solar composition. The inferred range of total pressure is similar to that predicted by dynamic models of the solar nebula for regions whose temperatures are in the range of silicate condensation temperatures. Alternatively, if departure from equilibrium condensation and/or non-representative sampling of condensates in the nebula occurred, the inferred range of total pressure could be smaller. Simple kinetic modeling of evaporation successfully reproduces observed chemical compositions of most inclusions from their inferred pre-evaporation compositions, suggesting that closed-system isotopic exchange processes did not have a significant effect on their isotopic compositions. Comparison of pre-evaporation compositions with observed ones indicates that $80 \%$ of the enrichment in refractory $\mathrm{CaO}+\mathrm{Al}_{2} \mathrm{O}_{3}$ relative to more volatile $\mathrm{MgO}+\mathrm{SiO}_{2}$ is due to initial condensation and $20 \%$ due to subsequent evaporation for both Type and Type B inclusions.

\section{ACKNOWLEDGMENTS}

We thank the Field Museum of Natural History for samples of the Allende meteorite, A. N. Krot for inclusions from the Efremovka meteorite, the National Museum of Natural History for a sample of the Leoville meteorite, M. Glascock of the University of Missouri Research Reactor for performing the chemical analyses by INAA, F. Ciesla for tutorials on nebular dynamics, and G. Eshel for help with statistics. This work was supported by NASA grants NAG5-11588 (to LG), NNG05GG00G (to LG), NNG05G187G (to MT), NNG06GF13G (to RNC) and W-19984 (to IDH). I. Hutcheon's work was performed under the auspices of the Dept. of Energy by the Univ. of California, Lawrence Livermore National Laboratory under Contract No. W-7405-Eng-48. T. Ding's work was supported by the Geological Survey of China under grant number 1212010561608. Prepared in part by LLNL under Contract DE-AC52-07NA27344

\section{REFERENCES}


Amelin Y., Grossman L., Krot A. N., Pestaj T., Simon S. B. and Ulyanov A. A. (2002) $\mathrm{U}-\mathrm{Pb}$ age of refractory inclusions from the $\mathrm{CV}$ carbonaceous chondrites Allende and Efremovka. In Lunar Planet. Sci. XXXIII, \#1151 (abstr.) [CD-ROM].

Anders E. and Grevesse N. (1989) Abundances of the elements: Meteoritic and solar. Geochim. Cosmochim. Acta 53, 197-214.

Berman R. G. (1983) A Thermodynamic Model for Multicomponent Melts, with Application to the System $\mathrm{CaO}-\mathrm{MgO}-\mathrm{Al}_{2} \mathrm{O}_{3}-\mathrm{SiO}_{2}$. Ph.D. dissertation, Univ. of British Columbia.

Chou C.-L., Baedecker P. A. and Wasson J. T. (1976) Allende inclusions: Volatileelement distribution and evidence for incomplete volatilization of presolar solids. Geochim. Cosmochim. Acta 40, 85-94.

Christophe Michel-Lévy M. (1968) Un chondre exceptionnel dans la météorite de Vigarano. Bull. Soc. fr. Minéral. Cristallogr. 91, 212-214.

Clayton R. N. and Mayeda T. K. (1963) The use of bromine pentafluoride in the extraction of oxygen from oxides and silicates for isotopic analysis. Geochim. Cosmochim. Acta 27, 43-52.

Clayton R. N. and Mayeda T. K. (1983) Oxygen isotopes in eucrites, shergottites, nakhlites, and chassignites. Earth Planet. Sci. Lett. 62, 1-6.

Clayton R. N., Onuma N., Grossman L. and Mayeda T. K. (1977) Distribution of the presolar component in Allende and other carbonaceous chondrites. Earth Planet. Sci. Lett. 34, 209-224.

Clayton R. N., Mayeda T. K., MacPherson G. J. and Grossman L. (1987) Oxygen and silicon isotopes in inclusions and chondrules from Vigarano. Lunar Planet. Sci. XVIII, 185-186 (abstr.).

Ding T. (2004) Analytical methods for silicon isotope determinations. In Handbook of Stable Isotope Analytical Techniques, vol. 1 (ed. P. A. De Groot). Elsevier, pp. 523-537.

Ding T., Wan D., Bai R., Zhang Z., Shen Y. and Meng R. (2005) Silicon isotope abundance ratios and atomic weights of NBS-28 and other reference materials. Geochim. Cosmochim. Acta 69, 5487-5494. 
Dowty E. and Clark J. R. (1973) Crystal structure refinement and optical properties of a $\mathrm{Ti}^{3+}$ fassaite from the Allende meteorite. Am. Mineral. 58, 230-242.

Ebel D. S. and Grossman L. (2000) Condensation in dust-enriched systems. Geochim. Cosmochim. Acta 64, 339-366.

Fedkin A. V. and Grossman L. (2006) The fayalite content of chondritic olivine: Obstacle to understanding the condensation of rocky material. In Meteorites and the Early Solar System II (eds. D. S. Lauretta and H. Y. McSween, Jr.). Univ. of Arizona Press, pp. 279-294.

Galy A., Young E. D., Ash R. D. and O’Nions R. K. (2000) The formation of chondrules at high gas pressures in the solar nebula. Science 290, 1751-1753.

Galy A., Belshaw N. S., Halicz L. and O’Nions R. K. (2001) High-precision measurement of magnesium isotopes by multiple-collector inductively coupled plasma mass spectrometry (MC-ICPMS). Int. J. Mass Spec. 208, 89-98.

Galy A., Yoffe O., Janney P. E., Williams R. W., Cloquet C., Alard O., Halicz L., Wadhwa M., Hutcheon I. D., Ramon E. and Carignan J. (2003) Magnesium isotope heterogeneity of the isotopic standard SRM980 and new reference materials for magnesium-isotope-ratio measurements. J. Anal. At. Spectrom. 18, 1352-1356.

Georg R. B., Halliday A. N., Schauble E. A. and Reynolds B. C. (2007) Silicon in the Earth's core. Nature 447, 1102-1106.

Grossman L. (1972) Condensation in the primitive solar nebula. Geochim. Cosmochim. Acta 36, 597-619.

Grossman L. (1980) Refractory inclusions in the Allende meteorite. Ann. Rev. Earth Planet. Sci. 8, 559-608.

Grossman L. and Fedkin A. V. (2003) $\mathrm{CaO}-\mathrm{MgO}-\mathrm{Al}_{2} \mathrm{O}_{3}-\mathrm{SiO}_{2}$ liquids: Chemical and isotopic effects of $\mathrm{Mg}$ and $\mathrm{Si}$ evaporation in a closed system of solar composition. Geochim. Cosmochim. Acta 67, 4205-4221.

Grossman L., Ebel D. S., Simon S. B., Davis A. M., Richter F. M. and Parsad N. M. (2000) Major element chemical and isotopic compositions of refractory inclusions in C3 
chondrites: The separate roles of condensation and evaporation. Geochim. Cosmochim. Acta 64, 2879-2894.

Grossman L., Ebel D. S. and Simon S. B. (2002) Formation of refractory inclusions by evaporation of condensate precursors. Geochim. Cosmochim. Acta 66, 145-161.

Grossman L., Beckett J. R., Fedkin A. V., Simon S. B. and Ciesla F. J. (2007a) Redox conditions in the solar nebula: Observational, experimental and theoretical constraints. In Oxygen in Earliest Solar System Materials and Processes (eds. G. J. MacPherson, D. W. Mittlefehldt, J. H. Jones and S. B. Simon). Revs. Mineral. Geochem. In press.

Grossman L., Simon S. B., Rai V. K., Thiemens M. H., Hutcheon I. D., Williams R. W., Galy A., Ding T., Clayton R. N. and Mayeda T. K. (2007b) Primordial compositions of refractory inclusions. Lunar Planet. Sci. XXXVIII, \#2172 (abstr.) [CD-ROM].

Knight K. B., Davis A. M., Kita N. T., Mendybaev R. A., Richter F. M. and Valley J. W. (2007) Silicon isotope fractionation in CAI-composition evaporation experiments. Meteoritics Planet. Sci. 42 Supp., A85 (abstr.).

Loss R. D., Lugmair G. W., MacPherson G. J. and Davis A. M. (1990) Isotope anomalies in Vigarano CAI's-hic et ubique. Lunar Planet. Sci. XXI, 718-719 (abstr.).

MacPherson G. J. and Davis A. M. (1993) A petrologic and ion microprobe study of a Vigarano Type B refractory inclusion: Evolution by multiple stages of alteration and melting. Geochim. Cosmochim. Acta 57, 231-243.

MacPherson G. J., Petaev M. I. and Krot A. N. (2004) Bulk compositions of CAIs and Al-rich chondrules: Implications of the reversal of the anorthite/forsterite condensation sequence at low nebular pressures. Lunar Planet. Sci. XXXV,\#1838 (abstr.)[CD-ROM].

MacPherson G. J., Simon S. B., Davis A. M., Grossman L. and Krot A. N. (2005) Calcium-aluminum-rich inclusions: Major unanswered questions. In Chondrites and the Protoplanetary Disk (eds. A. N. Krot, E. R. D. Scott and B. Reipurth). APS Conference Series, pp. 225-250.

McKeegan K. D. and Leshin L. A. (2001) Stable isotope variations in extraterrestrial materials. In Stable Isotope Geochemistry, Revs. Mineral. Geochem. 43 (eds. J. W. Valley and D. R. Cole). Mineral. Soc. Amer., Washington, D.C., pp. 279-318. 
Molini-Velsko C. (1983) Isotopic composition of silicon in meteorites. Ph.D. dissertation. The University of Chicago.

Molini-Velsko C., Mayeda T. K. and Clayton R. N. (1986) Isotopic composition of silicon in meteorites. Geochim. Cosmochim. Acta 50, 2719-2726.

Niederer F. R. and Papanastassiou D. A. (1984) Ca isotopes in refractory inclusions. Geochim. Cosmochim. Acta 48, 1279-1293.

Notsu K., Onuma N., Nishada N. and Nagasawa H. (1978) High temperature heating of the Allende meteorite. Geochim. Cosmochim. Acta 42, 903-907.

Petaev M. I. and Wood J. A. (1998) The CWPI model of nebular condensation: Effects of pressure on the condensation sequence. Meteoritics Planet. Sci. 33 Supp., A122 (abstr.).

Prombo C. A. and Lugmair G. W. (1986) Search for correlated isotope effects in Allende CAI's. Lunar Planet. Sci. XVII, 685-686 (abstr.).

Prombo C. A., Hashimoto A., Birck J. L., Lugmair G. W. and Grossman L. (1987) Search for correlated isotopic effects in Allende CAIs: II. Comparison with mineralogical data. Lunar Planet. Sci. XVIII, 804-805 (abstr.).

Richter F. M., Davis A. M., Ebel D. S. and Hashimoto A. (2002) Elemental and isotopic fractionation of Type B calcium-, aluminum-rich inclusions: Experiments, theoretical considerations, and constraints on their thermal evolution. Geochim. Cosmochim. Acta 66, 521-540.

Richter F. M., Janney P. E., Mendybaev R. A., Davis A. M. and Wadhwa M. (2005) On the temperature dependence of the kinetic isotope fractionation of Type B CAI-like melts during evaporation. Lunar Planet. Sci. XXXVI, \#2124 (abstr.)[CD-ROM].

Ruder S. P. and Pollack J. B. (1991) The dynamical evolution of the protosolar nebula. Astrophys J. 375, 740-760.

Simon S. B. and Grossman L. (2004) A preferred method for the determination of bulk compositions of coarse-grained refractory inclusions and some implications of the results. Geochim. Cosmochim. Acta 68, 4237-4248. 
Simon S. B., Davis A. M. and Grossman L. (1999) Origin of compact Type A refractory inclusions from CV3 carbonaceous chondrites. Geochim. Cosmochim. Acta 63, 12331248.

Simon S. B., Grossman L., Hutcheon I. D., Williams R. W., Galy A., Fedkin A. V., Clayton R. N. and Mayeda T. K. (2004) Determination of primordial refractory inclusion compositions. Lunar Planet. Sci. XXXV,\#1684 (abstr.) [CD-ROM].

Sylvester P. J., Grossman L. and MacPherson G. J. (1992) Refractory inclusions with unusual chemical compositions from the Vigarano carbonaceous chondrite. Geochim. Cosmochim. Acta 56, 1343-1363.

Tipper E. T., Galy A. and Bickle M. J. (2006) Riverine evidence for a fractionated reservoir of $\mathrm{Ca}$ and $\mathrm{Mg}$ on the continents: Implications for the oceanic Ca cycle. Earth Planet. Sci. Lett. 247, 267-279

Wark D. A. and Lovering J. F. (1977) Marker events in the early evolution of the solar system: Evidence from rims on Ca-Al-rich inclusions in carbonaceous chondrites. In Proc. Lunar Sci. Conf. $8^{\text {th }}$. Pegamon, pp. 95-112.

Wood J. A. (1967) Olivine and pyroxene compositions in Type II carbonaceous chondrites. Geochim. Cosmochim. Acta 31, 2095-2108.

Yoneda S. and Grossman L. (1995) Condensation of $\mathrm{CaO}-\mathrm{MgO}-\mathrm{Al}_{2} \mathrm{O}_{3}-\mathrm{SiO}_{2}$ liquids from cosmic gases. Geochim. Cosmochim. Acta 59, 3413-3444.

\section{FIGURE CAPTIONS}

Figure 1. Measurements of the magnesium isotopic compositions of CAIs performed in Livermore and Cambridge are usually in excellent agreement with one another, but two samples appear to be internally heterogeneous. Two-sigma uncertainties are shown when larger than the data points.

Figure 2. Oxygen isotopic data for CAIs measured in this work. All data plot very close to the CCAM line, indicating that there are no FUN inclusions among this suite of objects.

Figure 3. $\delta^{29} \mathrm{Si}$ vs $\delta^{30} \mathrm{Si}$, relative to NBS-28, of all inclusions in this study for which both were measured. The line drawn has the mean slope and intercept of the two least-squares 
regression lines through the new measurements (filled circles), whose typical $1 \sigma$ error bars are also shown. Some data from Molini-Velsko (1983; open circles) were also used here.

Figure 4. Condensation paths plotted in $\mathrm{SiO}_{2}-\mathrm{MgO}$ space for systems of solar composition at relatively (a) low and (b) high $P^{\text {tot }}$; and for systems enriched in OC dust by a factor of ten relative to solar composition at relatively (c) low and (d) high $P^{\text {tot }}$. Heavy arrows on paths point in the direction of decreasing temperature. Abbreviations: cor-corundum $\left(\mathrm{Al}_{2} \mathrm{O}_{3}\right)$; hib-hibonite $\left(\mathrm{CaAl}_{12} \mathrm{O}_{19}\right)$; gross-grossite $\left(\mathrm{CaAl}_{4} \mathrm{O}_{7}\right)$; mel-melilite; pv-perovskite; sp-spinel; cpx-clinopyroxene; an-anorthite; fo-forsterite; sap-sapphirine $\left(\mathrm{Mg}_{2} \mathrm{Al}_{4} \mathrm{SiO}_{10}\right)$; liq-CMAS liquid.

Figure 5. Best estimates of the bulk $\mathrm{SiO}_{2}$ and $\mathrm{MgO}$ contents (bold symbols) of all inclusions in this study, and their possible variations (plain symbols) due to nonrepresentative sampling in the laboratory. Filled symbols with fields circumscribed by solid curves-Type A inclusions; open symbols with fields circumscribed by dotted curves -Type Bs. Condensation paths calculated for a system of solar composition at relatively (a) low $P^{\text {tot }}$ and (b) high $P^{\text {tot }}$ are shown for reference.

Figure 6. The same data points as in Figure 5 compared to condensation paths calculated for a system enriched by a factor of ten in OC dust relative to gas compared to solar composition at relatively (a) low $P^{\text {tot }}$ and (b) high $P^{\text {tot }}$. Symbols as in Figure 5. Sample numbers omitted for clarity.

Figure 7. Isotopic mass fractionation of $\mathrm{Si}, \mathrm{F}_{\mathrm{Si}}$, plotted against that of $\mathrm{Mg}, \mathrm{F}_{\mathrm{Mg}}$, both relative to mean solar system matter, of all (a) Type A and (b) Type B inclusions in this study. Data compiled by Grossman et al. (2000) are shown for reference. Error bars are $1 \sigma$.

Figure 8. Fractions of $\mathrm{Mg}$ and $\mathrm{Si}$ evaporated for all inclusions in this work whose $\mathrm{F}_{\mathrm{Mg}}$ and $\mathrm{F}_{\mathrm{Si}}$ are both positive. Filled symbols are for Type $\mathrm{A}$ inclusions and open symbols are for Type Bs.

Figure 9. Initial, pre-evaporation bulk chemical compositions of the inclusions of this study, compared to condensation paths for systems of solar composition at relatively (a) low $P^{\text {tot }}$ and (b) high $P^{\text {tot }}$. Symbols as in Figure 5.

Figure 10. Initial, pre-evaporation bulk chemical compositions of the inclusions of this study, compared to condensation paths for a system enriched by a factor of ten in OC 
dust relative to gas compared to solar composition at relatively (a) low $P^{\text {tot }}$ and (b) high $P^{\text {tot }}$. Symbols as in Figure 5.

Figure 11. Computed curves tracing evaporative losses of $\mathrm{MgO}$ and $\mathrm{SiO}_{2}$ from preevaporation compositions that come closest to the specific observed compositions from which the former were inferred. The numbers are the absolute temperatures used to calculate the evaporation curves shown. For each inclusion, the open symbol indicates the pre-evaporation composition, and the filled symbol of the same shape indicates the observed composition.

Figure 12. Observed (a) and pre-evaporation (b) compositions of refractory inclusions are enriched in $\mathrm{CaO}+\mathrm{Al}_{2} \mathrm{O}_{3}$ relative to $\mathrm{MgO}+\mathrm{SiO}_{2}$ compared to solar abundances. About $80 \%$ of the enrichment in $\mathrm{CaO}+\mathrm{Al}_{2} \mathrm{O}_{3}$ relative to $\mathrm{MgO}+\mathrm{SiO}_{2}$ is due to initial condensation, and $20 \%$ is due to subsequent evaporation. Symbols as in Figure 9. 
Table 1. Chemical compositions of inclusions used in this work and not previously published ( $w \mathrm{t} \%$ unless otherwise indicated).

\begin{tabular}{|c|c|c|c|c|c|c|}
\hline Inclusion & TS32 & TS34 & F11 & E49 & E56 & E60 \\
\hline Type & CTA & B1 & CTA & CTA & CTA & Fo-bearing B \\
\hline Meteorite & Allende & Allende & Allende & Efremovka & Efremovka & Efremovka \\
\hline Method & $\mathrm{MR}^{1}$ & MR & MR & MR & MR & INAA \\
\hline $\mathrm{Na}_{2} \mathrm{O}(\mathrm{ppm})$ & n.d. & n.d. & n.d. & n.d. & n.d. & $640 \pm 6$ \\
\hline $\mathrm{MgO}$ & 5.73 & 10.74 & 5.76 & 3.71 & 5.47 & $16.82 \pm 0.06$ \\
\hline $\mathrm{Al}_{2} \mathrm{O}_{3}$ & 28.88 & 32.23 & 31.16 & 31.34 & 37.47 & $18.49 \pm 0.05$ \\
\hline $\mathrm{SiO}_{2}$ & 25.71 & 26.55 & 25.38 & 25.51 & 21.33 & $39.19^{2}$ \\
\hline $\mathrm{CaO}$ & 38.10 & 28.28 & 36.72 & 38.77 & 34.38 & $24.7 \pm 0.1$ \\
\hline $\mathrm{TiO}_{2}$ & 1.58 & 2.20 & 0.98 & 0.67 & 1.36 & $0.42 \pm 0.01$ \\
\hline $\mathrm{V}_{2} \mathrm{O}_{3}(\mathrm{ppm})$ & n.d. & n.d. & n.d. & n.d. & n.d. & $196 \pm 1$ \\
\hline $\mathrm{MnO}(\mathrm{ppm})$ & n.d. & n.d. & n.d. & n.d. & n.d. & $19.2 \pm 0.4$ \\
\hline $\mathrm{FeO}(\mathrm{ppm})$ & n.d. & n.d. & n.d. & n.d. & n.d. & $2973 \pm 57$ \\
\hline
\end{tabular}

${ }^{1} \mathrm{MR}$ : modal recombination. ${ }^{2}$ by difference. n.d.: not determined. 
Table 2. Mg-, O-, and Si-isotopic compositions measured for this study (\%o).

\begin{tabular}{|c|c|c|c|c|c|c|c|c|c|c|c|c|c|}
\hline Sample & Meteorite & $\begin{array}{l}\text { Incl. } \\
\text { Type }\end{array}$ & $\begin{array}{l}\delta^{26} \mathrm{Mg} \\
\text { LLNL }\end{array}$ & $\begin{array}{l}\delta^{25} \mathrm{Mg} \\
\text { LLNL }\end{array}$ & $\begin{array}{l}\delta^{26} \mathrm{Mg} \\
\text { Camb. }\end{array}$ & $\begin{array}{l}\delta^{25} \mathrm{Mg} \\
\text { Camb. }\end{array}$ & $\begin{array}{c}\delta^{18} \mathrm{O} \\
\mathrm{UCSD} \\
\end{array}$ & $\begin{array}{c}\delta^{17} \mathrm{O} \\
\mathrm{UCSD}\end{array}$ & $\begin{array}{c}\delta^{18} \mathrm{O} \\
\mathrm{UC} \\
\end{array}$ & $\begin{array}{c}\delta^{17} \mathrm{O} \\
\mathrm{UC} \\
\end{array}$ & $\begin{array}{c}\delta^{30} \mathrm{Si} \\
\text { Beijing }\end{array}$ & $\begin{array}{c}\delta^{29} \mathrm{Si} \\
\text { Beijing }\end{array}$ & $\begin{array}{c}\delta^{30} \mathrm{Si} \\
\mathrm{UC}\end{array}$ \\
\hline TS32 & $\mathrm{AL}$ & CTA & $\begin{array}{l}18.29 \\
\pm 0.31\end{array}$ & $\begin{array}{c}8.52 \\
\pm 0.20\end{array}$ & $\begin{array}{l}16.18 \\
\pm 0.05\end{array}$ & $\begin{array}{c}7.57 \\
\pm 0.05\end{array}$ & - & - & -7.3 & -11.0 & 2.42 & 1.29 & 2.52 \\
\hline E13 & $\mathrm{EF}$ & CTA & $\begin{array}{l}-0.80 \\
\pm 0.06 \\
\end{array}$ & $\begin{array}{l}-1.16 \\
\pm 0.05\end{array}$ & $\begin{array}{l}-0.36 \\
\pm 0.22 \\
\end{array}$ & $\begin{array}{l}-0.90 \\
\pm 0.11\end{array}$ & - & - & -34.5 & -37.4 & - & - & -1.02 \\
\hline E49A & $\mathrm{EF}$ & CTA & - & - & $\begin{array}{c}5.79 \\
\pm 0.07\end{array}$ & $\begin{array}{c}2.17 \\
\pm 0.05\end{array}$ & $\begin{array}{l}-3.14 \\
\pm 0.08\end{array}$ & $\begin{array}{l}-8.60 \\
\pm 0.17\end{array}$ & - & - & 1.62 & 0.82 & - \\
\hline E49 & $\mathrm{EF}$ & CTA & $\begin{array}{l}11.94 \\
\pm 0.20\end{array}$ & $\begin{array}{c}5.41 \\
\pm 0.07\end{array}$ & - & - & $\begin{array}{l}-0.95 \\
\pm 0.06\end{array}$ & $\begin{array}{l}-6.52 \\
\pm 0.05\end{array}$ & - & - & 2.30 & 1.17 & - \\
\hline E55 & $\mathrm{EF}$ & CTA & - & - & $\begin{array}{c}5.09 \\
\pm 0.34\end{array}$ & $\begin{array}{c}1.35 \\
\pm 0.23\end{array}$ & $\begin{array}{l}-4.41 \\
\pm 0.08\end{array}$ & $\begin{array}{l}-7.40 \\
\pm 0.06\end{array}$ & - & - & 3.74 & 1.95 & - \\
\hline E56 & $\mathrm{EF}$ & CTA & $\begin{array}{c}7.34 \\
\pm 0.73\end{array}$ & $\begin{array}{c}2.43 \\
\pm 0.19\end{array}$ & $\begin{array}{c}7.27 \\
\pm 0.15\end{array}$ & $\begin{array}{c}2.46 \\
\pm 0.10\end{array}$ & $\begin{array}{l}-7.16 \\
\pm 0.02\end{array}$ & $\begin{array}{c}-10.69 \\
\pm 0.03\end{array}$ & - & - & 1.66 & 0.81 & - \\
\hline E62 & $\mathrm{EF}$ & CTA & $\begin{array}{c}3.42 \\
\pm 0.33\end{array}$ & $\begin{array}{l}-1.25 \\
\pm .011\end{array}$ & - & - & $\begin{array}{l}-7.86 \\
\pm 0.04\end{array}$ & $\begin{array}{l}-12.40 \\
\pm 0.07\end{array}$ & - & - & 5.41 & 2.79 & - \\
\hline TS33 & $\mathrm{AL}$ & B1 & $\begin{array}{c}8.59 \\
\pm 0.15\end{array}$ & $\begin{array}{c}3.83 \\
\pm 0.07\end{array}$ & $\begin{array}{c}9.18 \\
\pm 0.13\end{array}$ & $\begin{array}{c}4.19 \\
\pm 0.01\end{array}$ & - & - & -19.4 & -21.5 & - & - & 2.82 \\
\hline TS34 & $\mathrm{AL}$ & B1 & $\begin{array}{l}11.89 \\
\pm 0.52\end{array}$ & $\begin{array}{c}5.63 \\
\pm 0.28\end{array}$ & - & - & - & - & - & - & - & - & - \\
\hline $3537-2$ & LE & B1 & $\begin{array}{c}7.54 \\
\pm 0.50 \\
\end{array}$ & $\begin{array}{c}3.20 \\
\pm 0.27 \\
\end{array}$ & $\begin{array}{c}7.56 \\
\pm 0.09 \\
\end{array}$ & $\begin{array}{c}3.32 \\
\pm 0.08\end{array}$ & - & - & -23.1 & -27.3 & - & - & 2.56 \\
\hline $\begin{array}{l}\text { F2 } \\
\text { (TS65) }\end{array}$ & $\mathrm{AL}$ & B2 & $\begin{array}{l}12.99 \\
\pm 0.10\end{array}$ & $\begin{array}{c}6.11 \\
\pm 0.05\end{array}$ & $\begin{array}{l}12.88 \\
\pm 0.08\end{array}$ & $\begin{array}{c}6.12 \\
\pm 0.03\end{array}$ & $\begin{array}{c}-15.98 \\
\pm 0.02\end{array}$ & $\begin{array}{c}-19.12 \\
\pm 0.07\end{array}$ & - & - & 4.10 & 2.11 & - \\
\hline E107 & $\mathrm{EF}$ & $\mathrm{A} / \mathrm{B}$ & $\begin{array}{c}4.83 \\
\pm 0.15 \\
\end{array}$ & $\begin{array}{c}1.86 \\
\pm 0.10 \\
\end{array}$ & $\begin{array}{c}5.17 \\
\pm 0.16 \\
\end{array}$ & $\begin{array}{c}2.12 \\
\pm 0.05 \\
\end{array}$ & $\begin{array}{l}-10.18 \\
\pm 0.05\end{array}$ & $\begin{array}{c}-14.38 \\
\pm 0.04 \\
\end{array}$ & - & - & 0.92 & 0.55 & - \\
\hline Golfball & $\mathrm{AL}$ & B & - & - & $\begin{array}{c}3.86 \\
\pm 0.11 \\
\end{array}$ & $\begin{array}{c}1.61 \\
\pm 0.06 \\
\end{array}$ & $\begin{array}{l}-22.10 \\
\pm 0.03\end{array}$ & $\begin{array}{l}-25.70 \\
\pm 0.05 \\
\end{array}$ & - & - & 0.49 & 0.39 & - \\
\hline E60 & $\mathrm{EF}$ & Fo-B & $\begin{array}{c}7.69 \\
\pm 0.35 \\
\end{array}$ & $\begin{array}{c}3.78 \\
\pm 0.12 \\
\end{array}$ & $\begin{array}{c}7.75 \\
\pm 0.07 \\
\end{array}$ & $\begin{array}{c}3.78 \\
\pm 0.03 \\
\end{array}$ & $\begin{array}{l}-21.06 \\
\pm 0.02 \\
\end{array}$ & $\begin{array}{l}-24.77 \\
\pm 0.09 \\
\end{array}$ & - & - & 2.91 & 1.56 & - \\
\hline
\end{tabular}


Stated uncertainties for $\mathrm{Mg}$ are $2 \sigma$. Uncertainties for Si isotopic data are $\pm 0.2 \%$ for $\delta^{29} \mathrm{Si}$ and $\pm 0.4 \%$ for $\delta^{30} \mathrm{Si}(2 \sigma)$. For the oxygen isotopic compositions, uncertainties on the UC data are $<0.1 \%$, and the stated uncertainties for the UCSD data are $1 \sigma$. LLNL: Lawrence Livermore National Laboratories. Camb.: Cambridge University. UCSD: University of California, San Diego. UC: University of Chicago. AL: Allende. EF: Efremovka. LE: Leoville. 
Table 3. Average compositions of melilite and fassaite in compact Type A inclusions.

\begin{tabular}{lcccc}
\hline & Melilite & s.d. & Fassaite & s.d. \\
\hline $\mathrm{MgO}$ & 3.37 & 1.05 & 6.70 & 0.66 \\
$\mathrm{Al}_{2} \mathrm{O}_{3}$ & 28.69 & 2.66 & 20.27 & 2.11 \\
$\mathrm{SiO}_{2}$ & 26.86 & 1.51 & 32.48 & 1.31 \\
$\mathrm{CaO}$ & 40.99 & 0.42 & 24.86 & 0.28 \\
$\mathrm{TiO}_{2}$ & 0.07 & 0.04 & 15.78 & 2.18 \\
$\mathrm{No}$ of inclusions & 8 & & 5 & \\
\hline
\end{tabular}

s.d.: standard deviation 


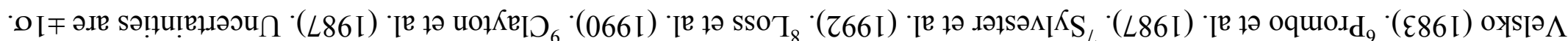

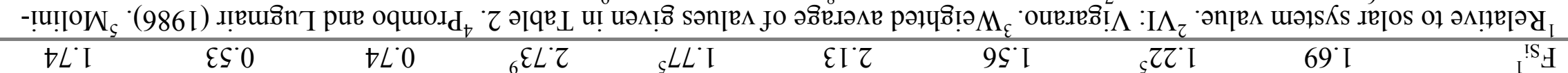

\begin{tabular}{|c|c|c|c|c|c|c|c|c|c|}
\hline${ }_{\varepsilon} Z 0^{\circ} 0 \mp 8 L^{\circ} \varepsilon$ & $\varepsilon 0^{\circ} 0 \mp\left[9^{\circ} \mathrm{I}\right.$ & ${ }_{\varepsilon} Z 0^{\circ} 0 \mp \angle 0^{\circ} Z$ & ${ }_{8} \varsigma^{\circ} 0 \mp 6 \cdot 6$ & $9^{\circ} 0 \mp 6^{\circ} \nabla$ & ${ }_{\varepsilon} Z 0^{\circ} 0 \mp Z I^{\circ} 9$ & ${ }_{\varepsilon} \succsim 0^{\circ} 0 \mp \mathrm{I} \varepsilon^{*} \mathcal{\varepsilon}$ & $\downarrow I^{\circ} 0 \mp \varepsilon 9^{\circ} \varsigma$ & ${ }_{\varepsilon} \mathrm{I}^{\circ} 0 \mp 8 \mathrm{I}^{\circ} \mathrm{t}$ & ${ }^{\circ} \mathrm{W}_{\mathrm{H}}$ \\
\hline $69^{\circ} \varepsilon \varepsilon$ & $8 \varsigma^{\circ} 0 \mathcal{E}$ & $68^{\circ}+\tau$ & $\tau 9^{\circ} \circ \tau$ & $9 I^{\circ} 0 \mathcal{E}$ & $88^{\circ} 87$ & $\nabla I^{\circ} L Z$ & $t \varepsilon^{\cdot} 9 \tau$ & $00^{\circ} 87$ & ${ }^{\tau} \mathrm{O} ! \mathrm{S}$ \\
\hline $19^{\circ} 97$ & $I \mathcal{E}^{\circ} I \mathcal{E}$ & $0 L^{\circ} \varsigma \mathcal{E}$ & $\tau L \bullet \dagger \varepsilon$ & $9 \varepsilon \cdot \tau \varepsilon$ & $\varepsilon t^{\circ} \varepsilon \varepsilon$ & $98^{\circ} \nabla \varepsilon$ & $\varepsilon \varsigma^{\circ} \nabla \varepsilon$ & $\nabla \varepsilon \cdot \nabla \varepsilon$ & ${ }^{\varepsilon} \mathrm{O}^{\mathrm{Z}} \mathrm{IV}$ \\
\hline$\varepsilon 9{ }^{\circ} \mathrm{I}$ & $\tau \varepsilon^{\cdot} \mathcal{E} I$ & 9II I & $\varsigma I^{\bullet} \mathcal{E} I$ & $98^{\circ}$ I I & $Z Z^{\circ} \mathrm{II}$ & $0 t^{\circ} 0 \mathrm{I}$ & $6 L^{\circ} \mathrm{II}$ & $6 t^{\circ} 0 \mathrm{I}$ & OฮิW \\
\hline$\angle 0^{\circ} I Z$ & $6 L^{\circ} \nabla Z$ & $97^{\circ} 87$ & I $S^{\circ} L Z$ & $\tau 9^{\circ} \varsigma 乙$ & $\angle t^{\circ} 9 Z$ & $09^{\circ} \angle Z$ & $\nabla \varepsilon^{*} L Z$ & $8 I^{\circ} L Z$ & $\mathrm{O}^{\mathrm{P}} \mathrm{P}$ \\
\hline$\forall \forall N I$ & yW & yW & ${ }_{L} \mathrm{VVNI}$ & yW & yW & yW & yW & 'dW & рочрәК \\
\hline G-OH & g & g & zg & zg & zg & Ig & Ig & Ig & $\partial \mathrm{d} \kappa_{\mathrm{L}}$ \\
\hline 实早 & $\mathrm{TV}$ & 크 & ${ }_{2} \mathrm{I} \Lambda$ & TV & TV & 百Т & TV & $\mathrm{TV}$ & 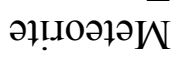 \\
\hline 09日 & IIRqJIOD & LOIG & 8-ยZ9I & (L9SL) LH & 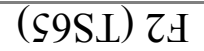 & $\tau-\angle \mathcal{E} \mathcal{E}$ & $\nabla \varepsilon S L$ & EESL & uo!̣n!つU \\
\hline
\end{tabular}

\begin{tabular}{|c|c|c|c|c|c|c|c|c|}
\hline $66^{\circ} z$ & $t L^{\cdot} I$ & $\varsigma I^{\prime} Z$ & $87^{\circ} \mathrm{I}$ & $60^{\circ} \mathrm{I}$ & $\varepsilon Z^{*} 0^{-}$ & ${ }_{\varsigma} E t^{*} I$ & ${ }_{\varepsilon} I^{*} I$ & ${ }_{I}^{!} \mathrm{S}_{\mathrm{H}}$ \\
\hline $90^{\circ} 0 \mp \varsigma Z^{\circ} I^{-}$ & ${ }_{\varepsilon} \subseteq 0^{\circ} 0 \mp \varsigma t^{*} \tau$ & $\mathcal{E} I^{\circ} 0 \mp \varsigma \mathcal{E}^{\cdot} \mathrm{I}$ & t0.0干It $\varsigma$ & $\varepsilon 0^{\circ} 0^{\prime} L^{\prime} \tau$ & ${ }_{\varepsilon} \varepsilon 0^{\circ} 0 \mp Z I^{*} I^{-}$ & ${ }_{t} \varepsilon^{*} I \mp 6{ }^{\circ} t$ & ${ }_{\varepsilon} \varepsilon 0^{\circ} 0 \mp \varepsilon 9^{\circ} L$ & ${ }^{8} \mathrm{~N}_{H}$ \\
\hline $09 \% 6 \mathrm{I}$ & $9 t^{\circ} 0 z$ & $\nabla \varepsilon \cdot \nabla \tau$ & $s 8^{\circ} 0 Z$ & $\varsigma \mathcal{E}^{*} I Z$ & $69^{\circ} \angle Z$ & t9'zz & $\varsigma 6.6 \mathrm{I}$ & ${ }^{2} \mathrm{O} ! \mathrm{S}$ \\
\hline$\angle 9^{\circ}$ It & $\varsigma Z^{\circ} 0 t$ & $\varepsilon 0^{\circ} 9 \varepsilon$ & $9 I^{\circ} 6 \varepsilon$ & $\varepsilon 0^{\circ} L \mathcal{E}$ & $\mathrm{II}^{\circ} S \mathcal{E}$ & $8 L^{\circ} L E$ & $09^{\circ} 8 \mathcal{\varepsilon}$ & ${ }^{\varepsilon} \mathrm{O}^{2} \mathrm{IV}$ \\
\hline$\varepsilon L \cdot S$ & $Z \nabla^{\circ} L$ & $I^{\circ} \mathrm{II}$ & $66^{\circ} 8$ & $0 \varepsilon^{\bullet} Z I$ & $0 t^{\circ} 6$ & $89 \%$ & $68^{\circ} 0 \mathrm{I}$ & OฮิW \\
\hline $66^{\circ} \tau \varepsilon$ & $\angle 8^{\circ} I E$ & $\varepsilon \varsigma^{\circ} 8 \tau$ & $00^{\circ} \mathrm{IE}$ & $\tau \varepsilon \cdot 6 \tau$ & $08^{\circ} \angle Z$ & $16 \cdot 62$ & $9 \varsigma^{\circ} 0 \varepsilon$ & $\mathrm{O}^{\mathrm{e}} \mathrm{O}$ \\
\hline$\forall \forall N I$ & צ' & $\forall \forall \mathrm{NI}$ & yW & $\forall \forall N I$ & $\forall \forall N I$ & УW & УW & рочю口 \\
\hline VLO & VLO & VLO & VLO & VLO & VLO & VLO & VLO & $\partial \mathrm{d} \kappa_{\mathrm{L}}$ \\
\hline 변 & 켜 & 켜 & 켜 & Н马 & 期 & $\mathrm{TV}$ & $\mathrm{TV}$ & 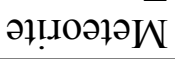 \\
\hline 29日 & 9S马 & Sऽ月 & 6†日 & 6⽢日 & EIG & (89SL) I IH & ZESL & uo!snโगUI \\
\hline
\end{tabular}

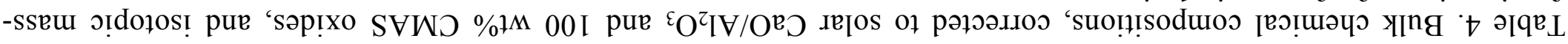




\begin{tabular}{|c|c|c|c|c|c|c|c|}
\hline $80^{\circ} \varsigma \mathcal{\varepsilon}$ & $90^{\circ} I \mathcal{E}$ & $69^{\circ} \varsigma 乙$ & $\mathrm{It} \varsigma \mathrm{c}$ & $\varepsilon 6^{\circ}$ I $\varepsilon$ & $\varepsilon 6^{\circ} 0 \varepsilon$ & $t z \cdot 6 z$ & ${ }^{2} \mathrm{O} ! \mathrm{S}$ \\
\hline$\angle \varepsilon^{\circ} \varepsilon \tau$ & $8 I^{\circ} 0 \mathcal{E}$ & $9 \tau^{\circ} \dagger \varepsilon$ & $\varepsilon \nabla^{\circ} L Z$ & $08^{\circ} 87$ & $\angle 0^{\circ} 6 Z$ & $\nabla \tau \cdot \tau \varepsilon$ & ${ }^{\varepsilon} \mathrm{O}^{\mathrm{Z}} \mathrm{IV}$ \\
\hline$\varsigma 0^{\circ} \mathcal{} \mathcal{}$ & $98^{\circ} \nabla \mathrm{I}$ & $\varepsilon 6^{\circ} \mathrm{ZI}$ & $t t^{\circ} \varsigma \tau$ & $9 t^{\circ} 9 \mathrm{I}$ & $66^{\circ} 9 \mathrm{I}$ & $66^{\circ} \mathrm{ZI}$ & Oฮิ \\
\hline $0 S^{\circ} 8 \mathrm{I}$ & $06^{\circ} \varepsilon z$ & $Z I^{\circ} L Z$ & $\varepsilon L^{\cdot} I Z$ & $08^{\circ} z z$ & $\mathrm{I} 0^{\circ} \varepsilon z$ & $\varepsilon \varsigma \varsigma^{*} \tau$ & $\mathrm{O}^{\mathfrak{B}} \mathrm{O}$ \\
\hline $\mathrm{g}-\mathrm{O}_{\mathrm{H}}$ & g & G & zg & zg & zg & Ig & $\partial \mathrm{d} \kappa_{\mathrm{L}}$ \\
\hline 期 & $\mathrm{TH}$ & 可寻 & $\mathrm{I} \Lambda$ & $\mathrm{TV}$ & $\mathrm{TV}$ & 日7 & әұ!ฺоәџәW \\
\hline 09日 & ІІеqJоD & LOIG & 8-๕Z9I & $(\angle 9 S L) \angle H$ & (ৎ9SL) Z & $z-\angle \mathcal{E} S \mathcal{E}$ & uo!̣snjouI \\
\hline
\end{tabular}

\begin{tabular}{|c|c|c|c|c|c|c|c|c|}
\hline $0 L^{\circ} 9 z$ & $\angle 0^{\circ} 0 \mathcal{E}$ & $00^{\circ} \varepsilon z$ & $\angle 0^{\circ} 8 Z$ & $\varsigma \tau^{\prime} \tau Z$ & $99^{\circ} z z$ & $90^{\circ} \nabla \tau$ & IS ${ }^{\circ} 0 Z$ & ${ }^{2} \mathrm{O} ! \mathrm{S}$ \\
\hline$\varsigma 0^{\circ} I \mathcal{E}$ & $\varsigma Z^{\circ} I \mathcal{E}$ & $9 I^{\circ} 8 \mathcal{E}$ & $99^{\circ} \varepsilon \mathcal{E}$ & $\varsigma I^{\circ} 9 \varepsilon$ & $\tau \mathcal{E}^{\circ} \varsigma \mathcal{\varepsilon}$ & $06^{\circ} \nabla \varepsilon$ & $\varepsilon \tau^{\circ} \nabla \varepsilon$ & ${ }^{\varepsilon} \mathrm{O}^{2} \mathrm{IV}$ \\
\hline $99^{\circ} \angle I$ & $\varsigma 6^{\circ} \varepsilon I$ & 29.8 & I9 I I & $66^{\circ} \mathrm{ZI}$ & $s 0 \circ \mathrm{I}$ & $\tau \nabla^{\circ} \varepsilon I$ & $9 I^{\circ} 8 \mathrm{I}$ & OฮิW \\
\hline $6 \varsigma^{\circ} \nabla \tau$ & $\varepsilon L^{\circ} \nabla \tau$ & $z \tau^{\circ} 0 \varepsilon$ & $99^{\circ} 97$ & $19^{\circ} 82$ & $\angle 6 \angle Z$ & $\varepsilon 9^{\circ} L Z$ & $0 I^{\circ} L Z$ & $\mathrm{O}^{\mathrm{e}} \mathrm{O}$ \\
\hline Ig & Ig & VID & VLO & VLO & VID & VLO & VLO & $\partial \mathrm{d} \kappa_{\mathrm{I}}$ \\
\hline $\mathrm{TV}$ & $\mathrm{TV}$ & 期 & 봉 & 扫 & 期 & $\mathrm{TV}$ & TV & әұцџоәџәК \\
\hline$\downarrow \varepsilon S L$ & $\varepsilon \varepsilon S L$ & 9S日 & ऽ९马 & 6†日 & V6t日 & (89SL) I I & ZESL & uo!sn[गU] \\
\hline
\end{tabular}

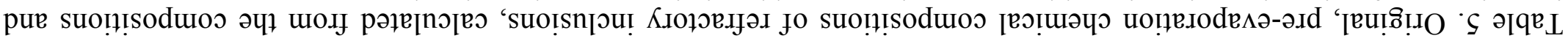




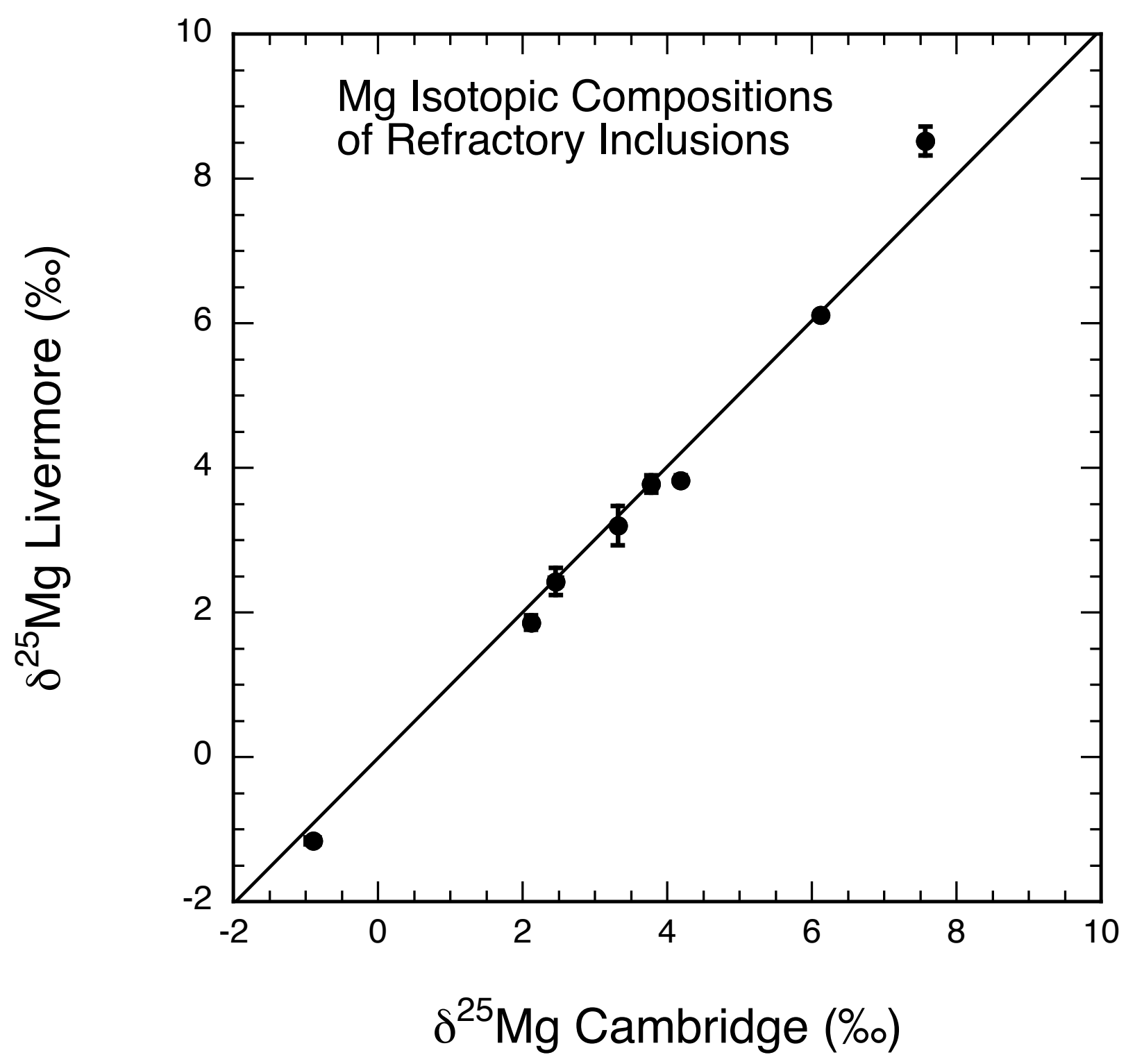

Fig. 1 


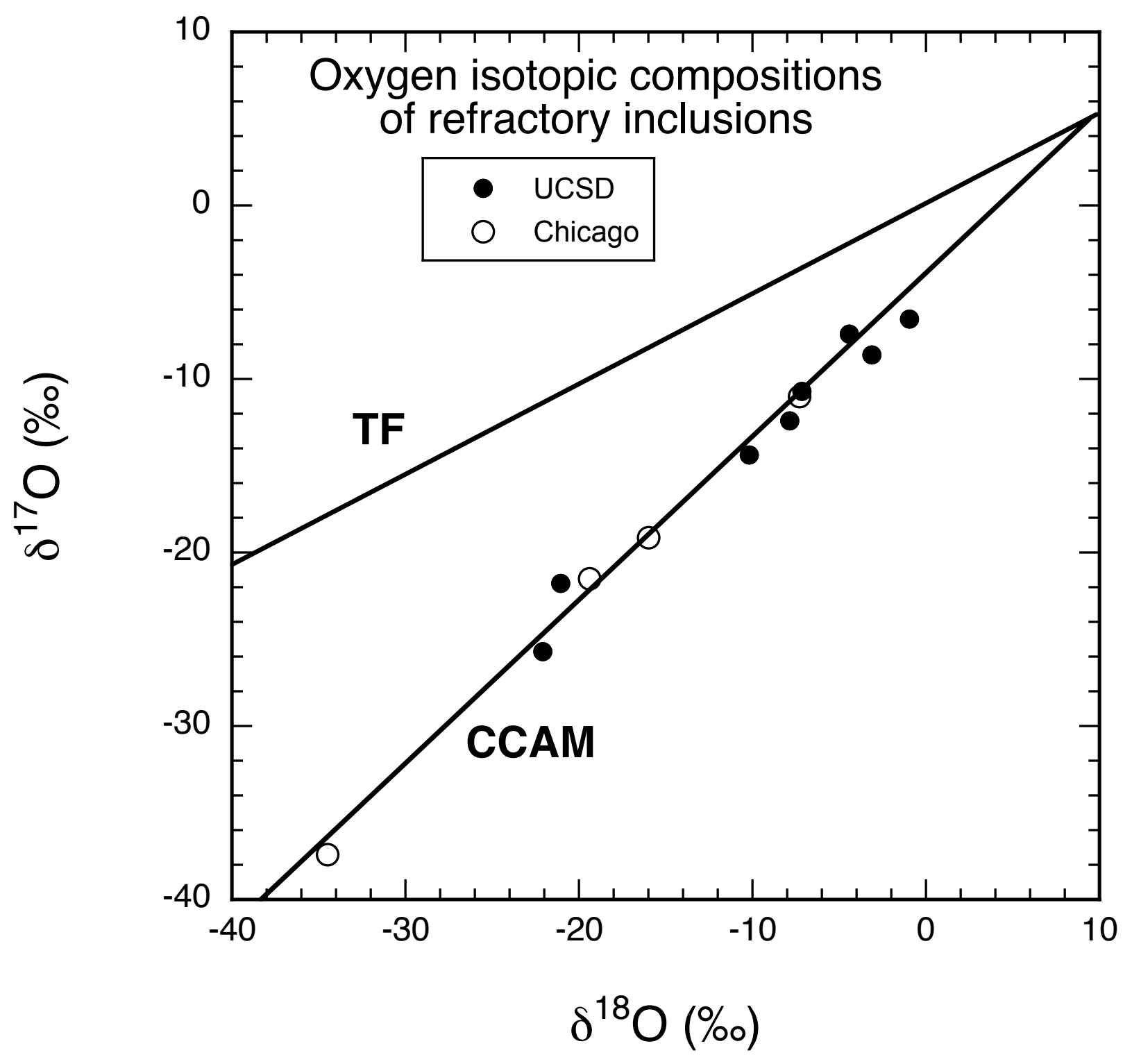

Fig. 2 


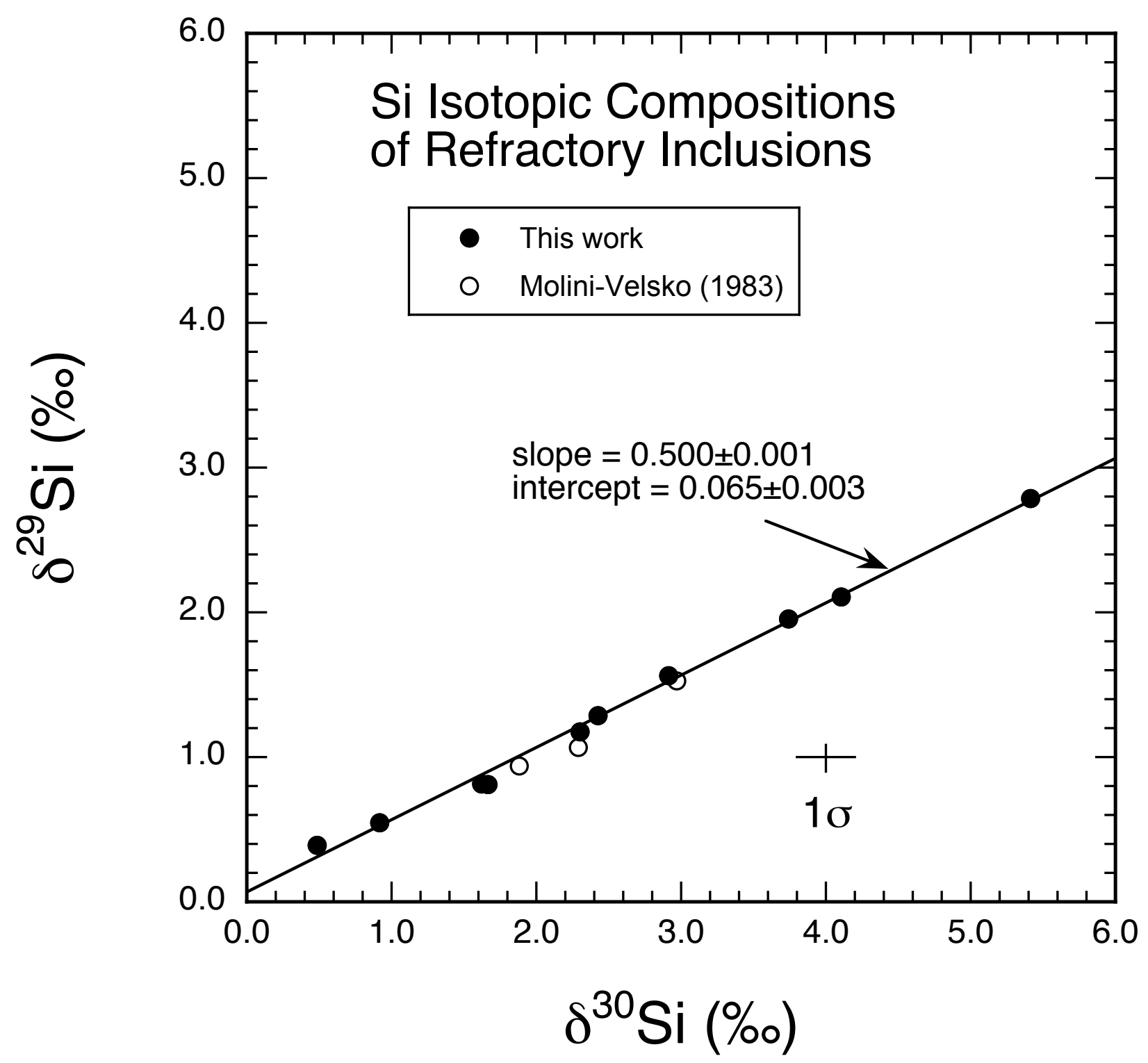

Fig. 3 


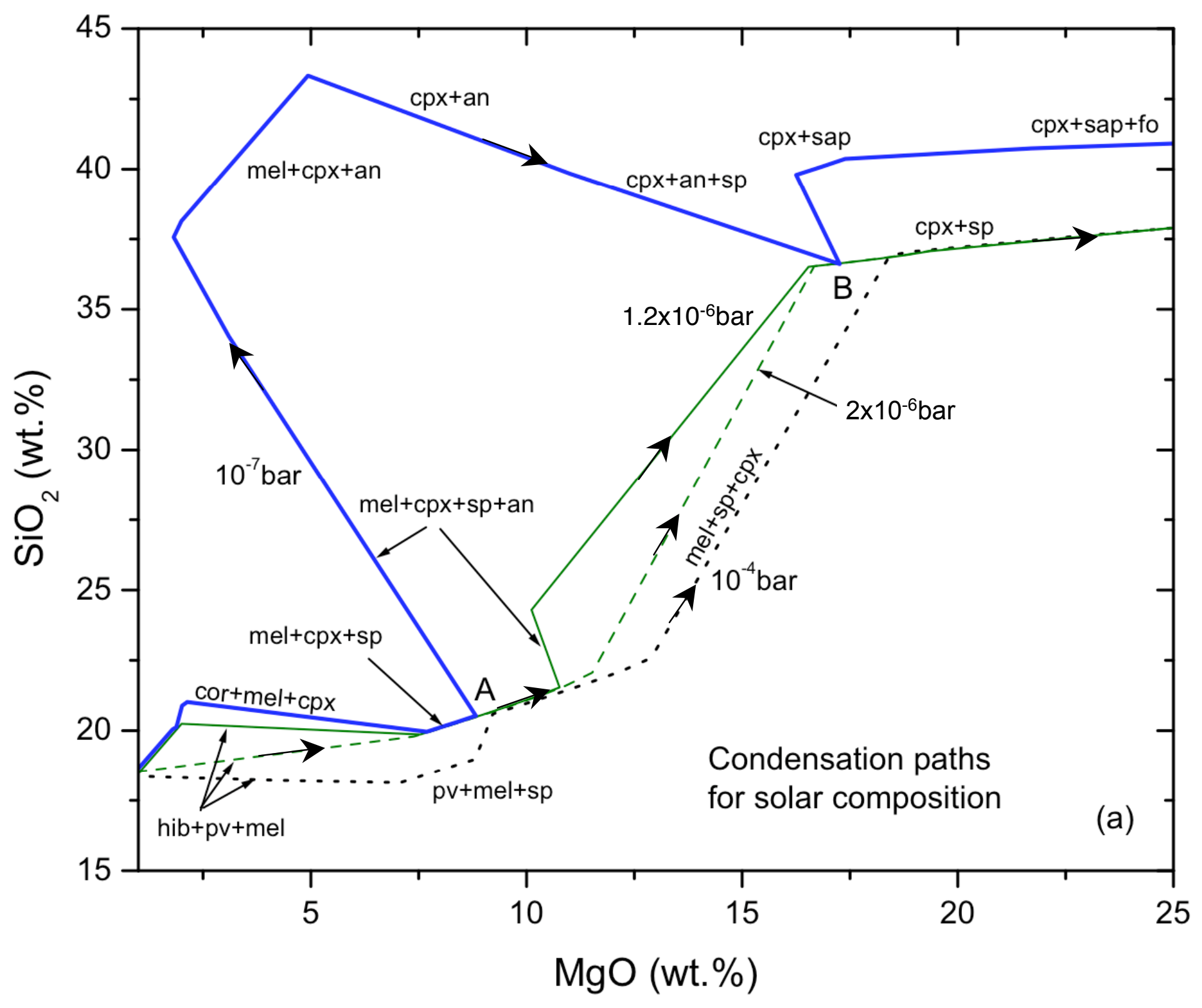




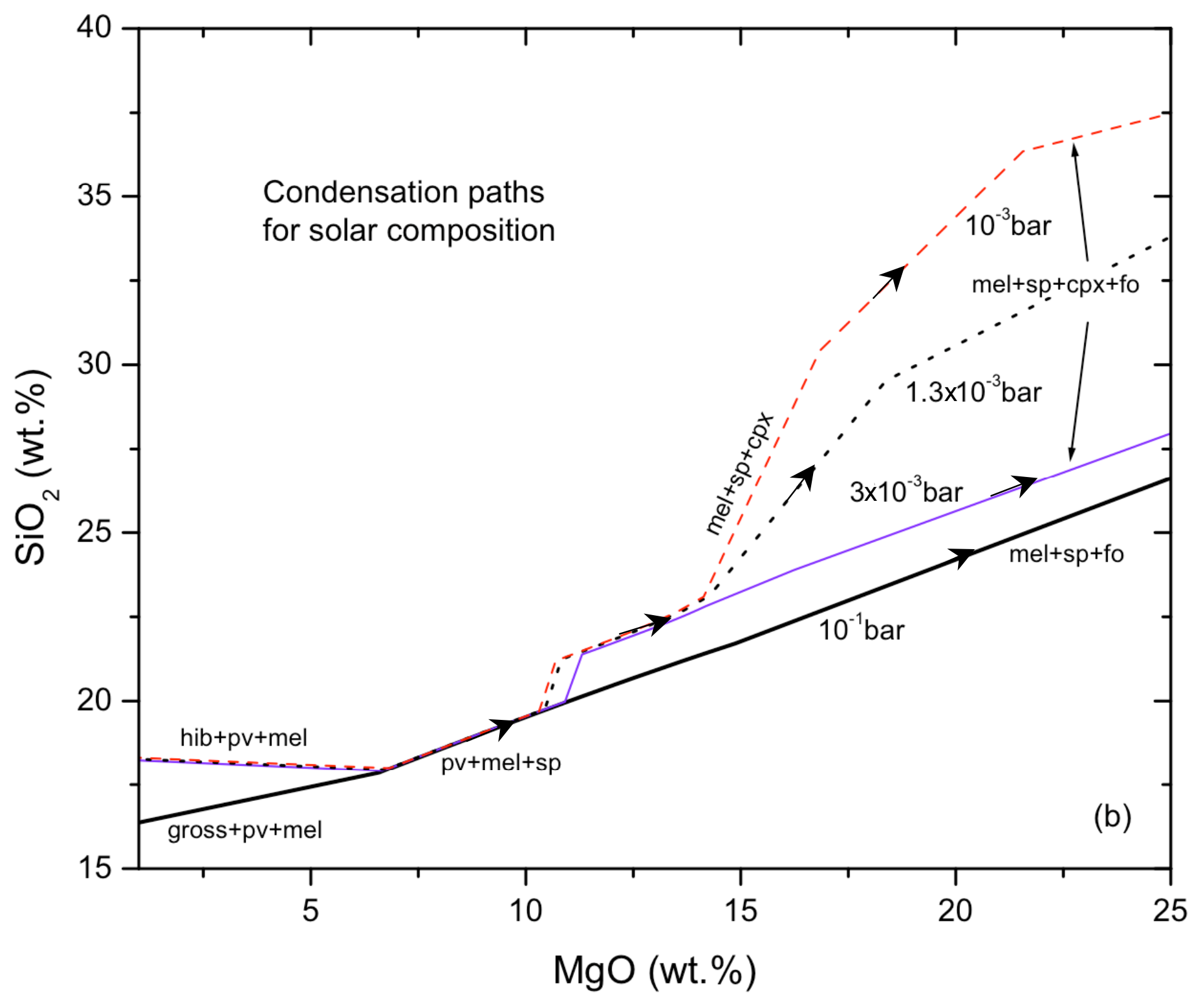




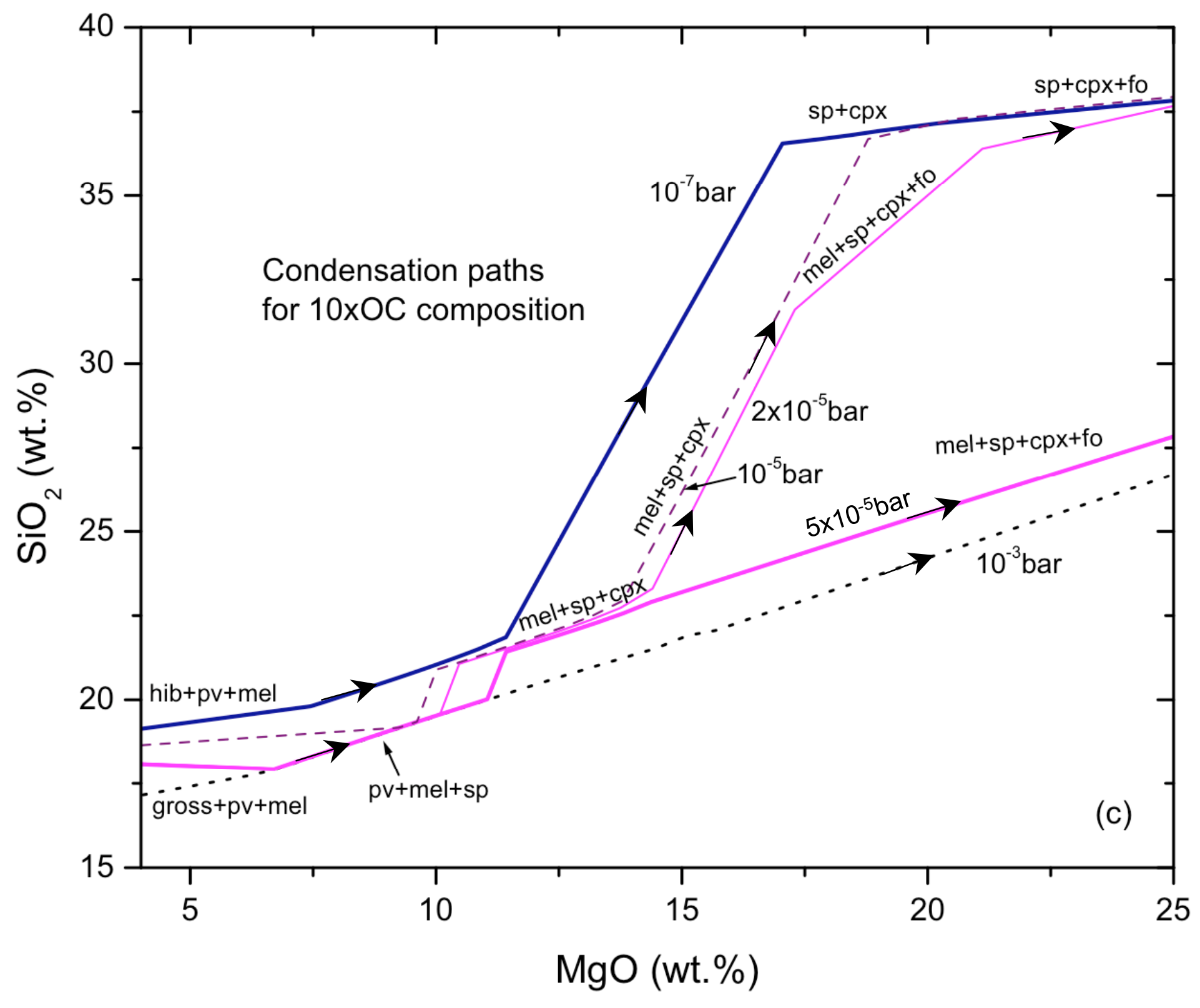




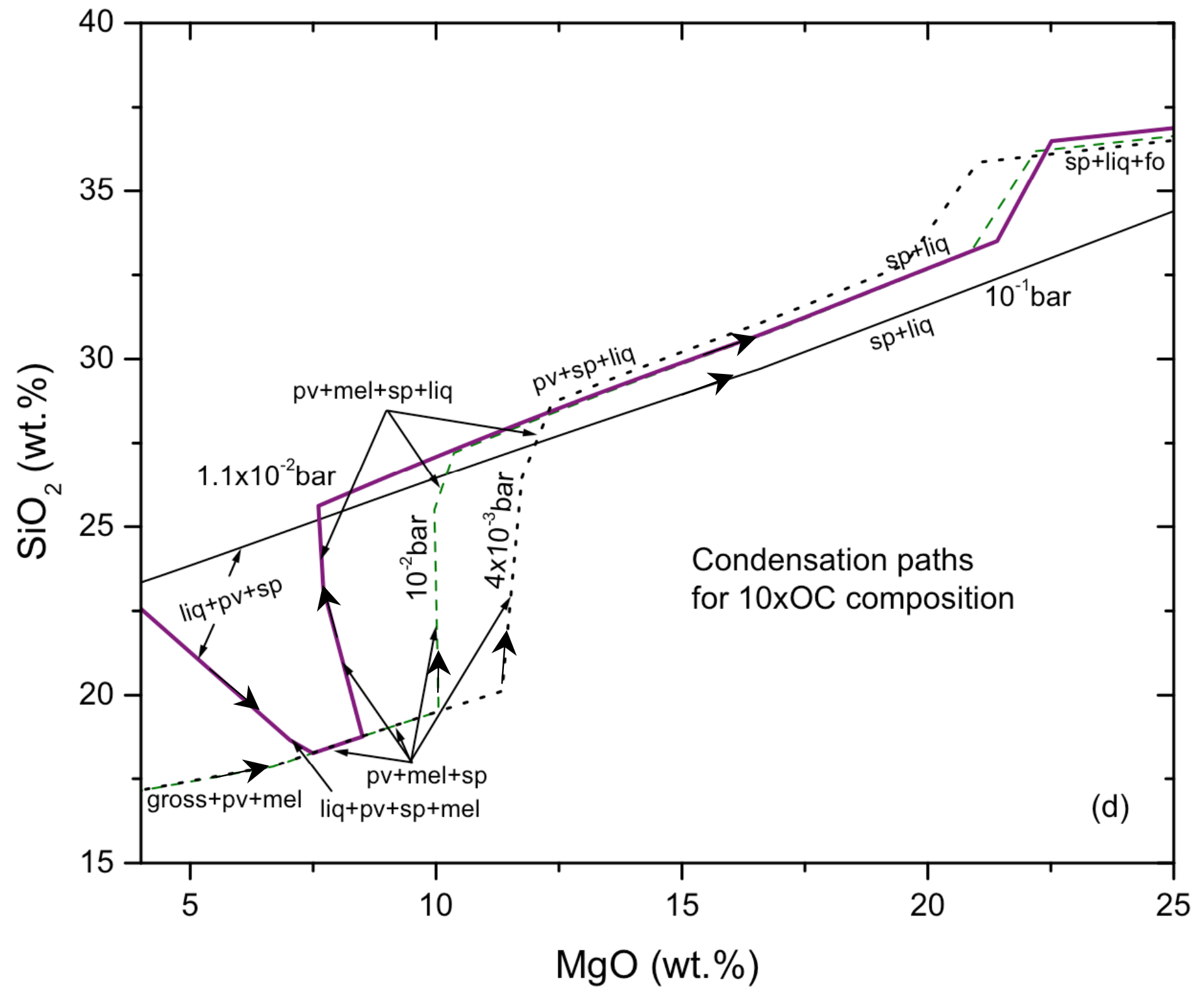




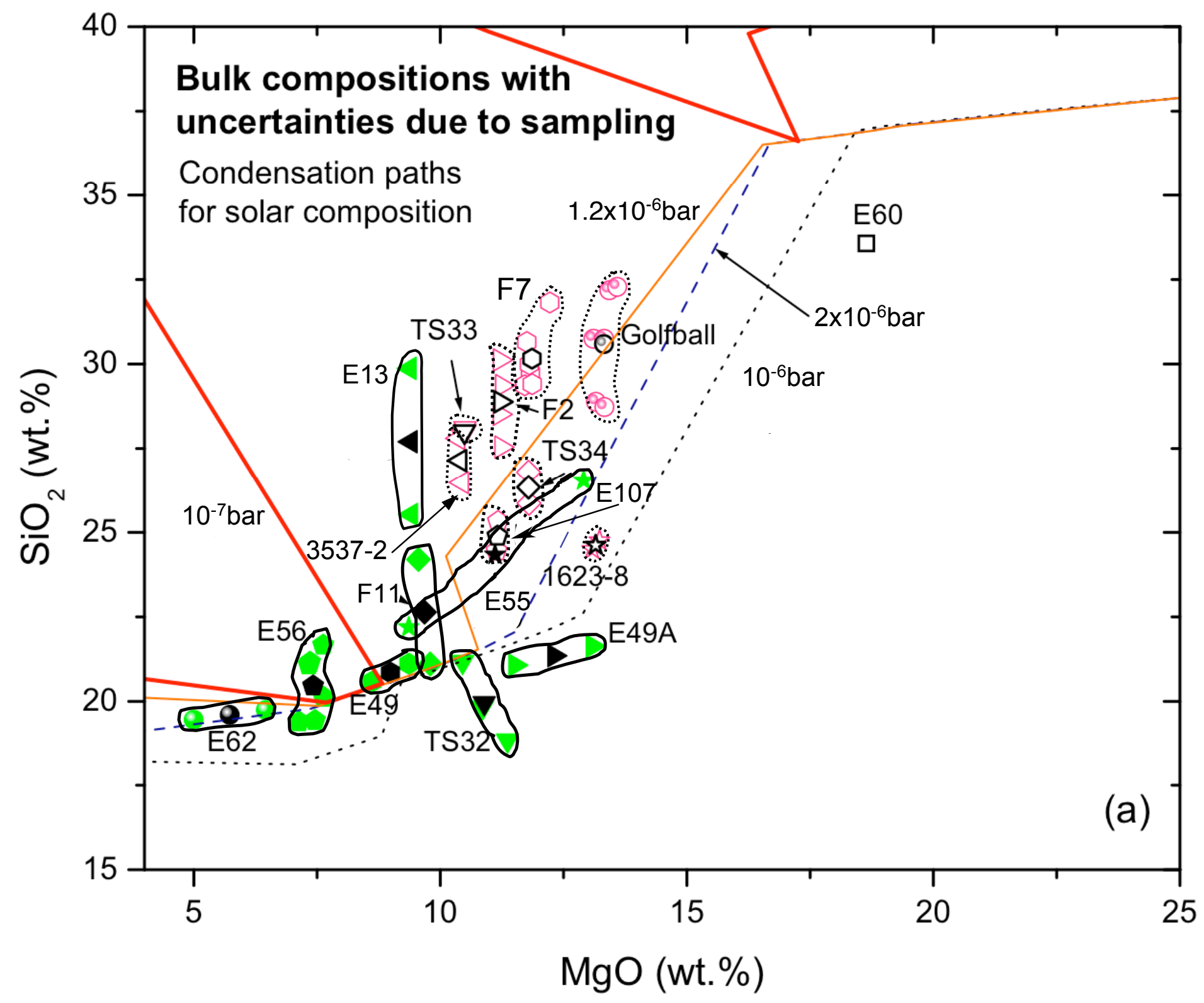




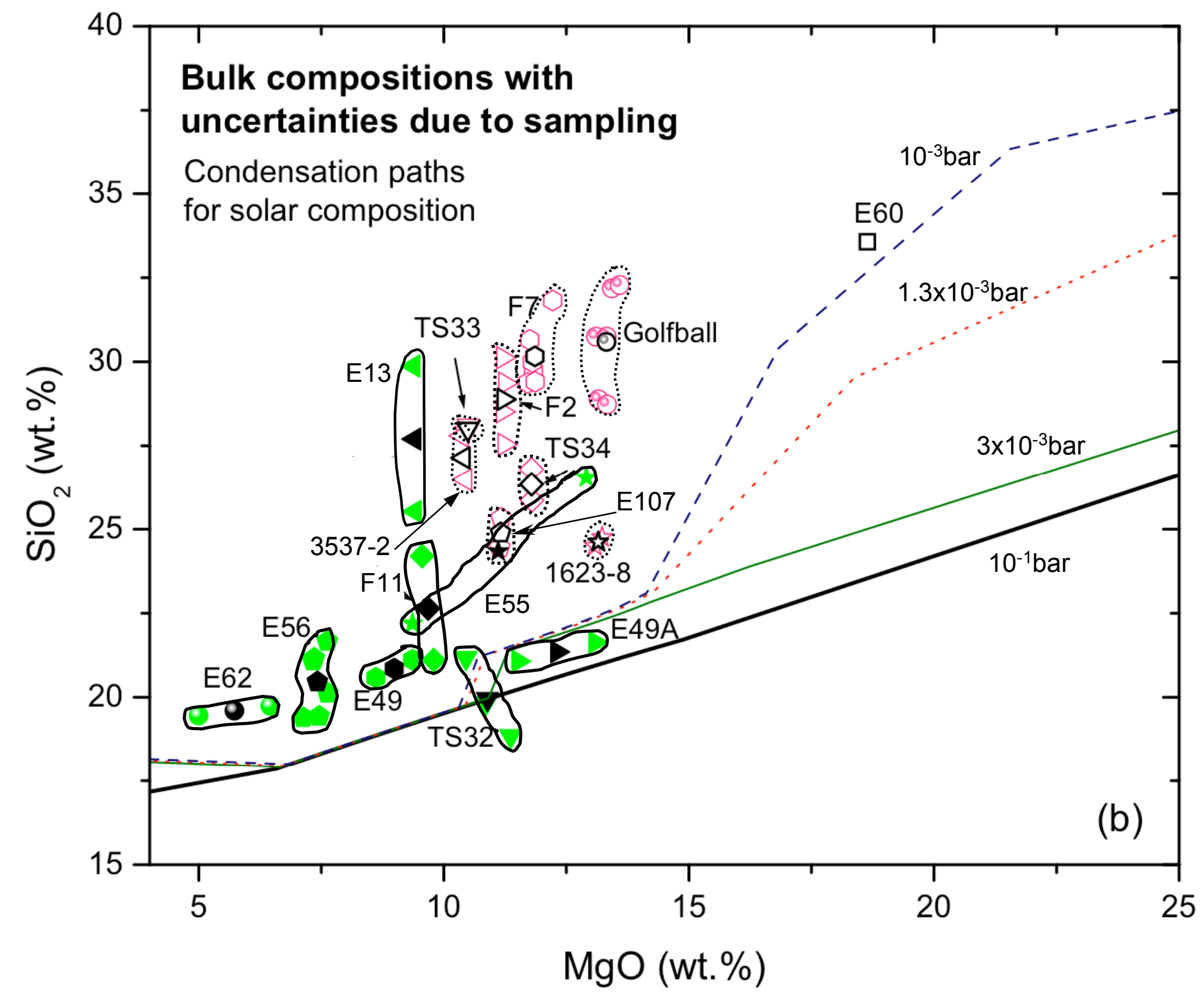




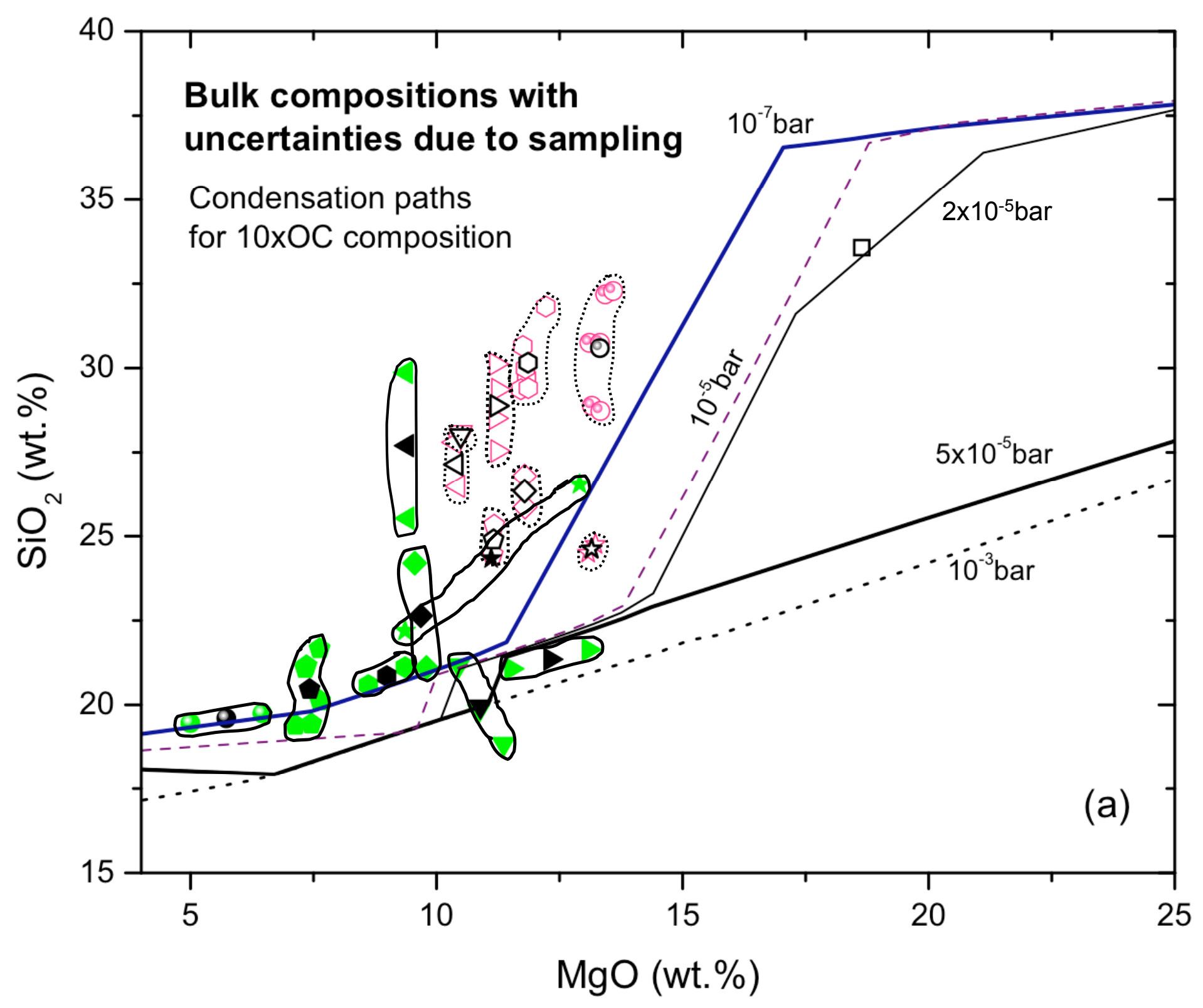




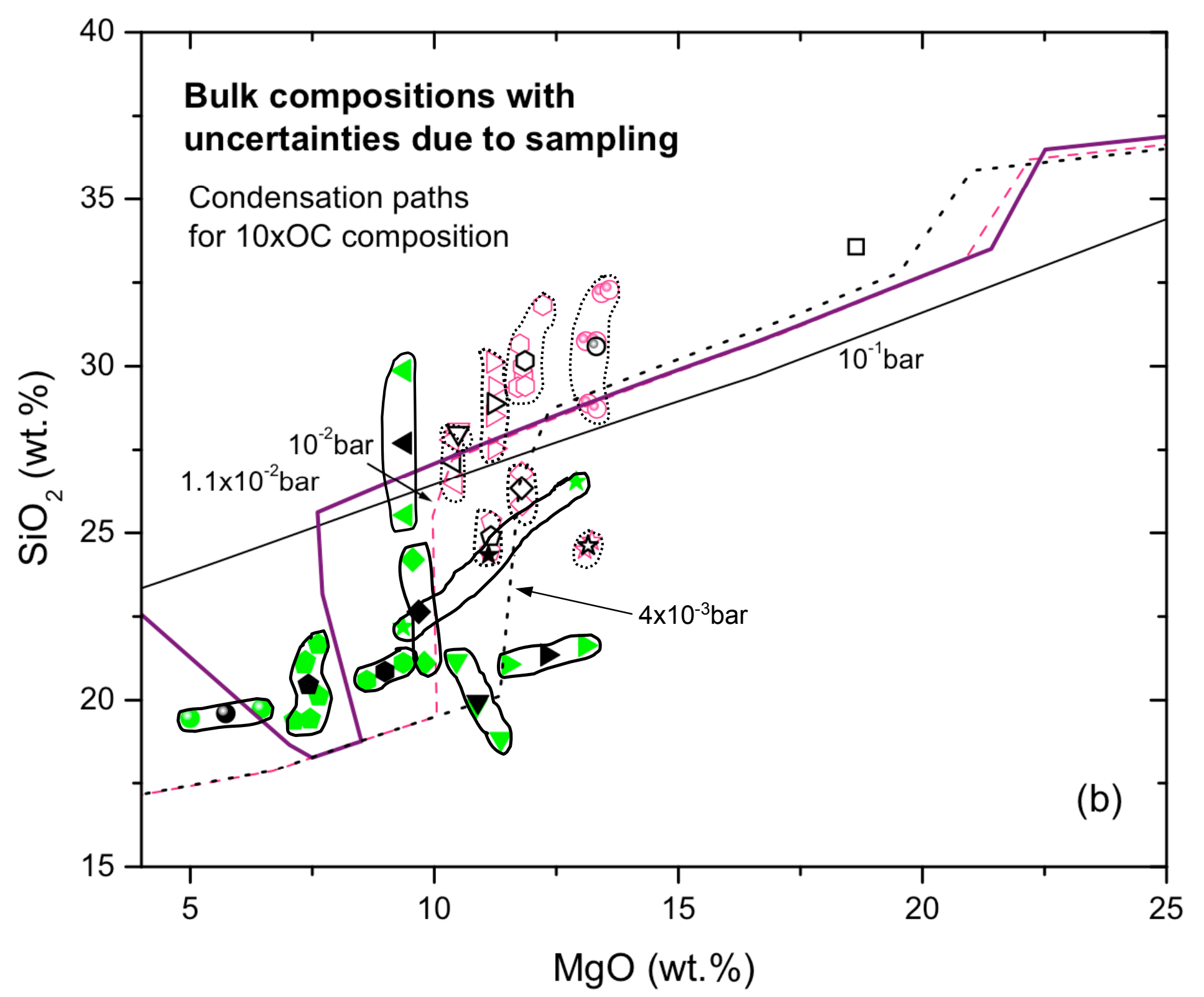




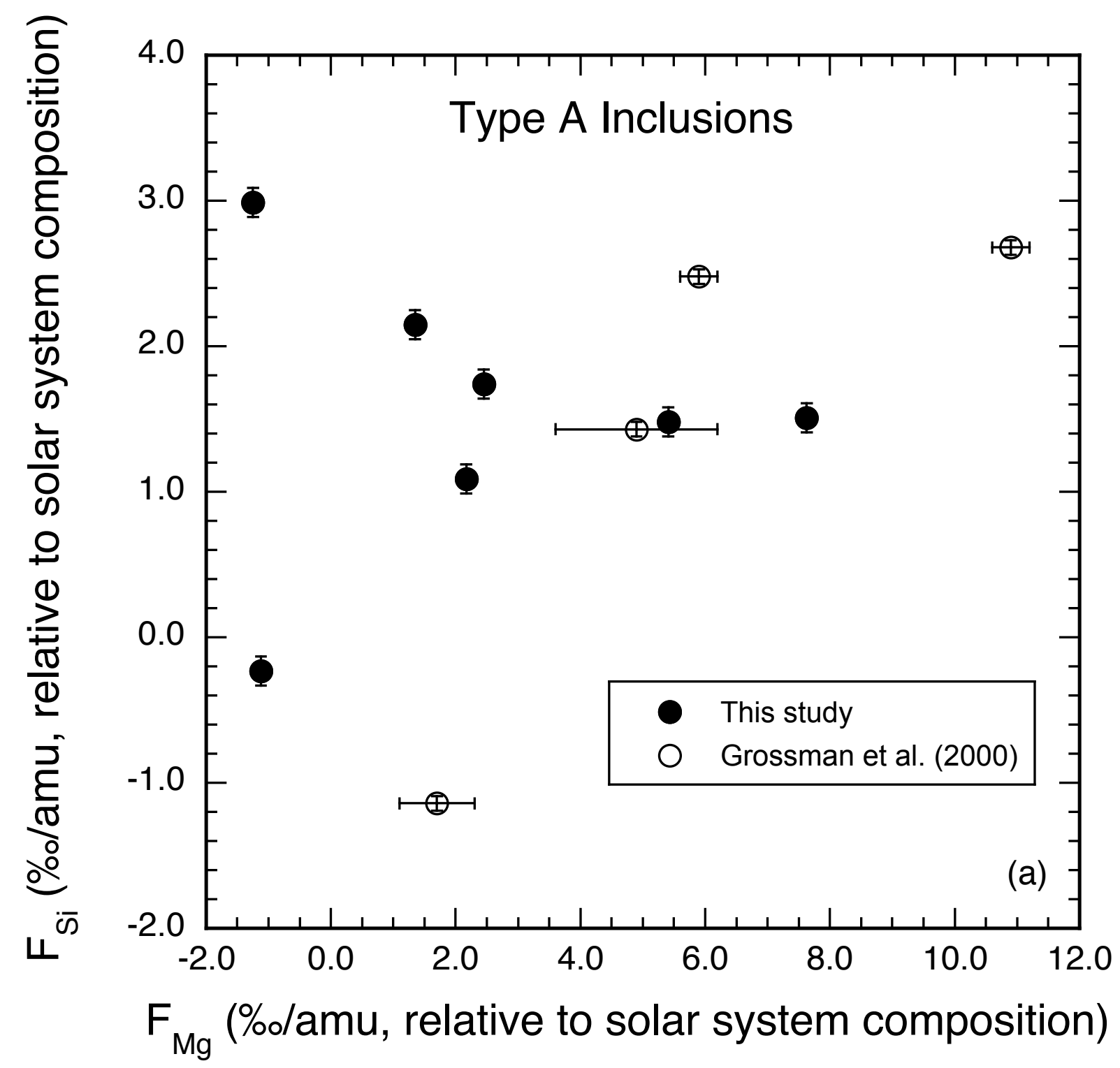

Fig. $7 a$ 


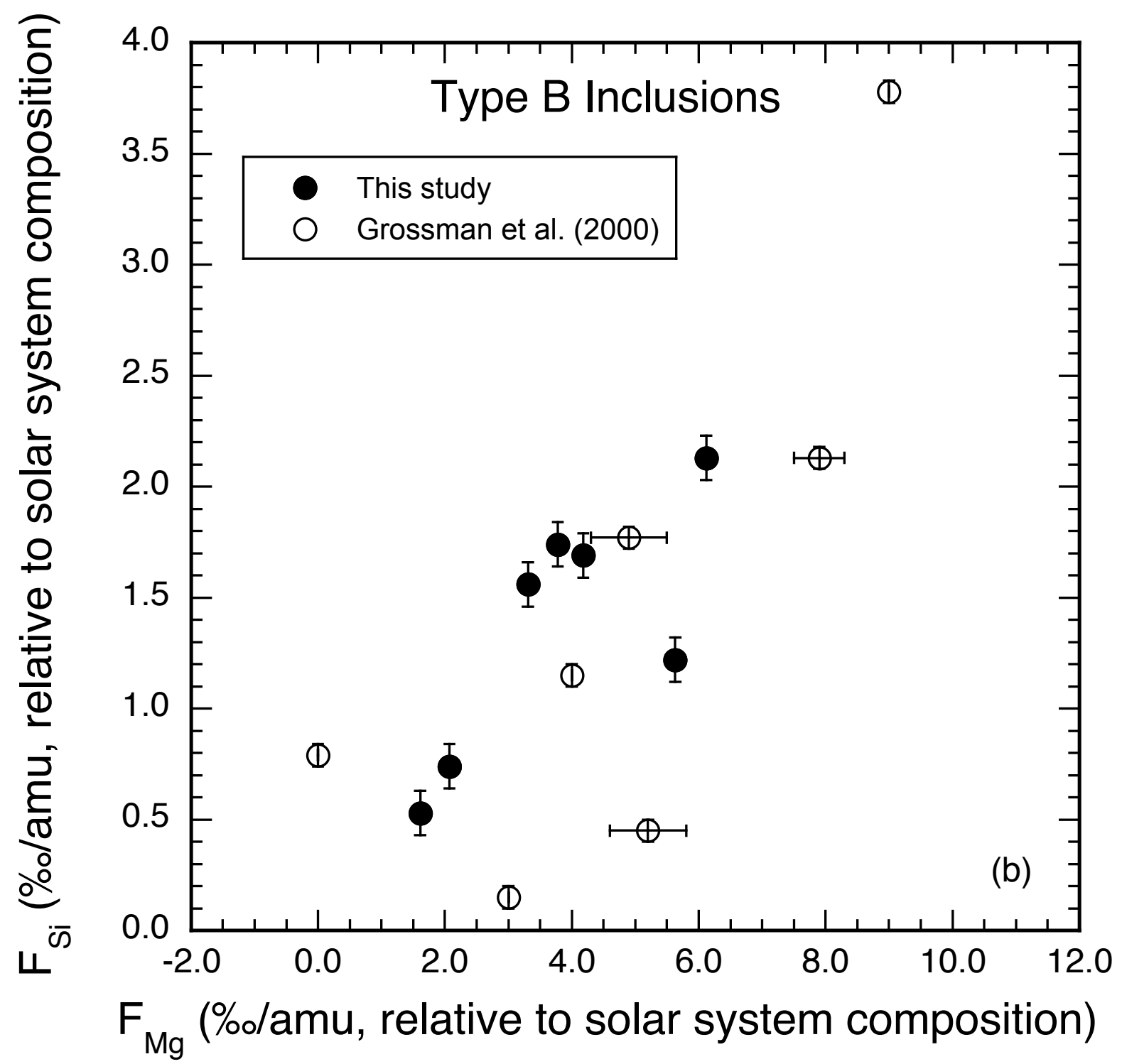

Fig. $7 b$ 


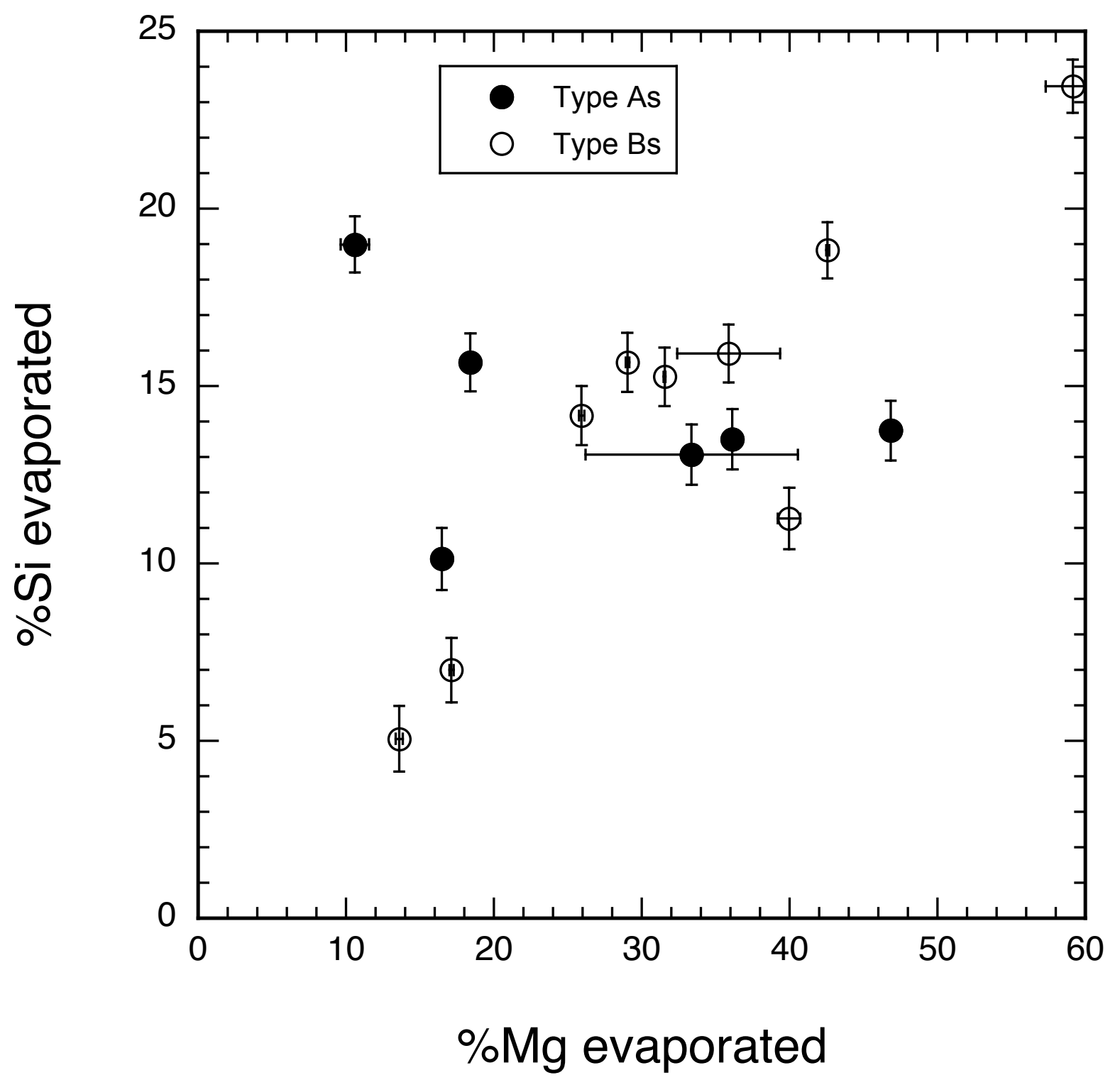

Fig. 8 


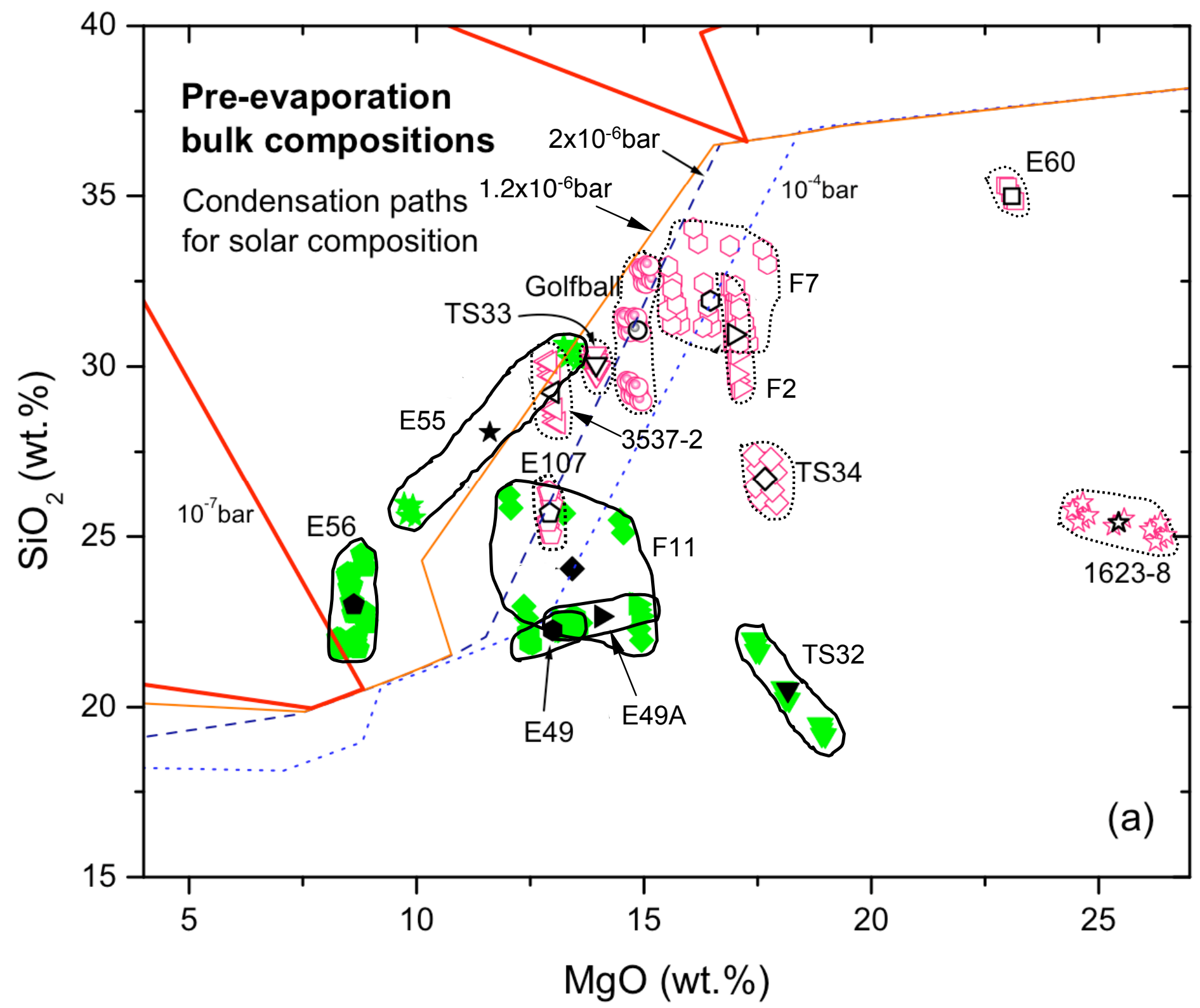




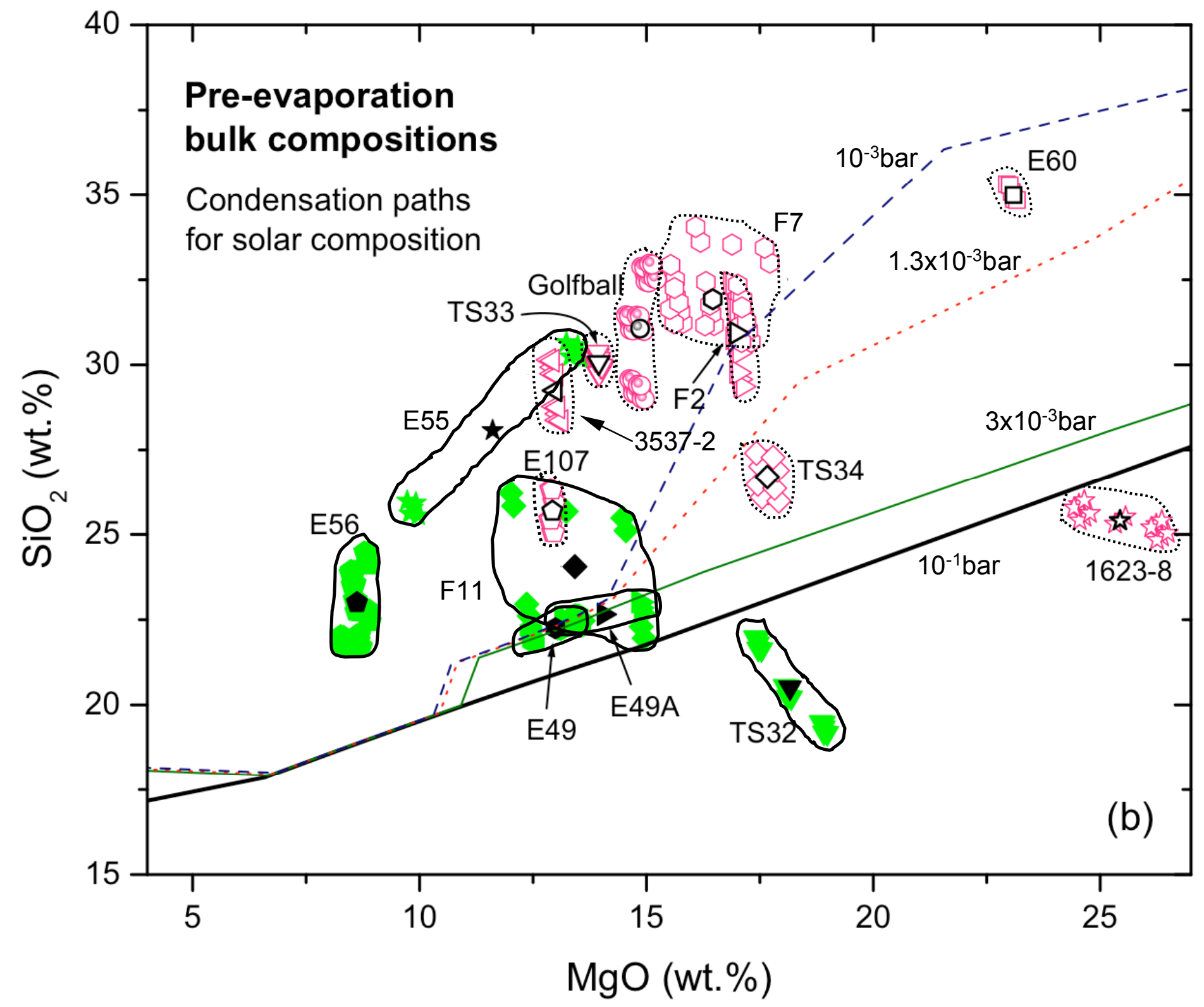




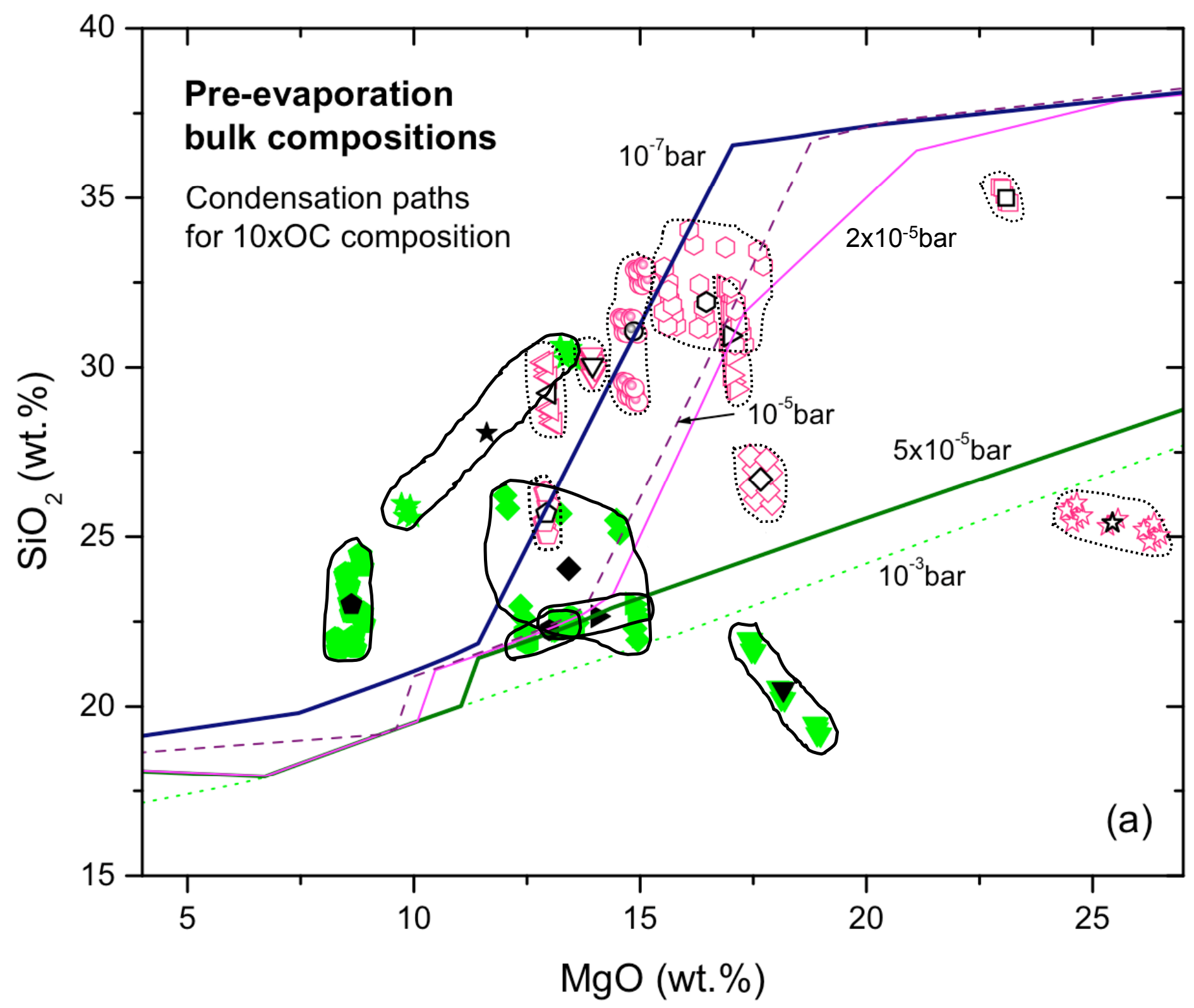




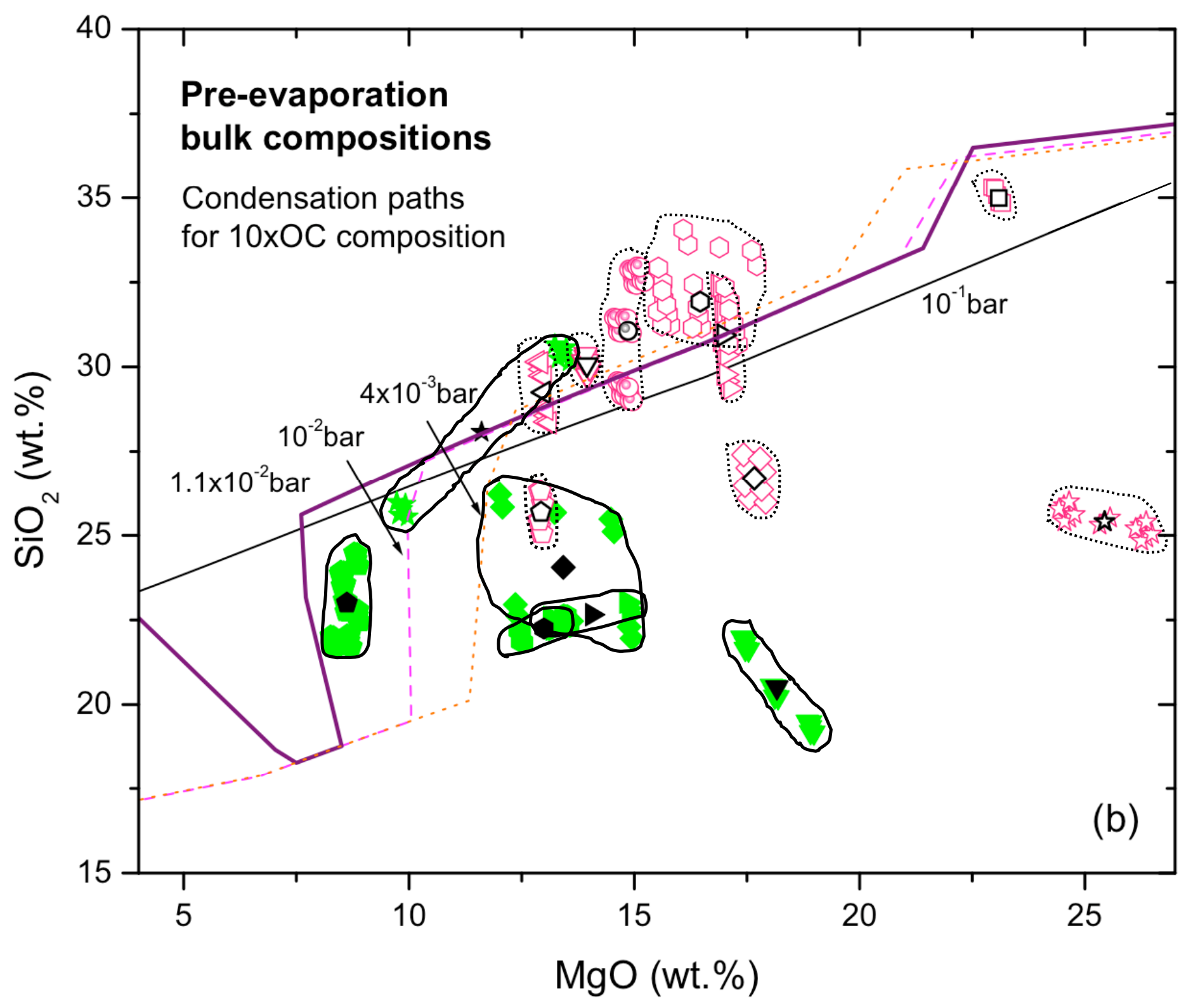




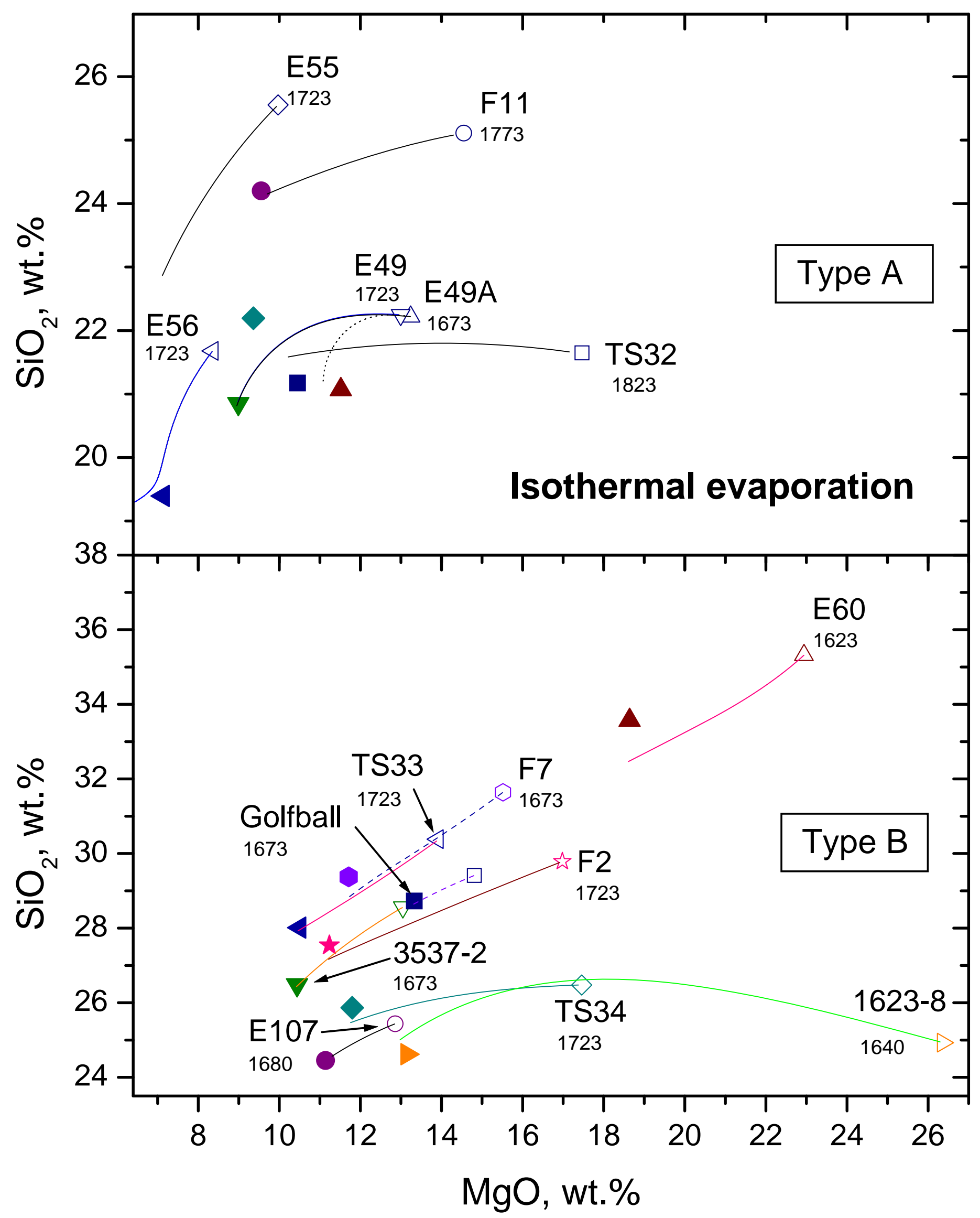




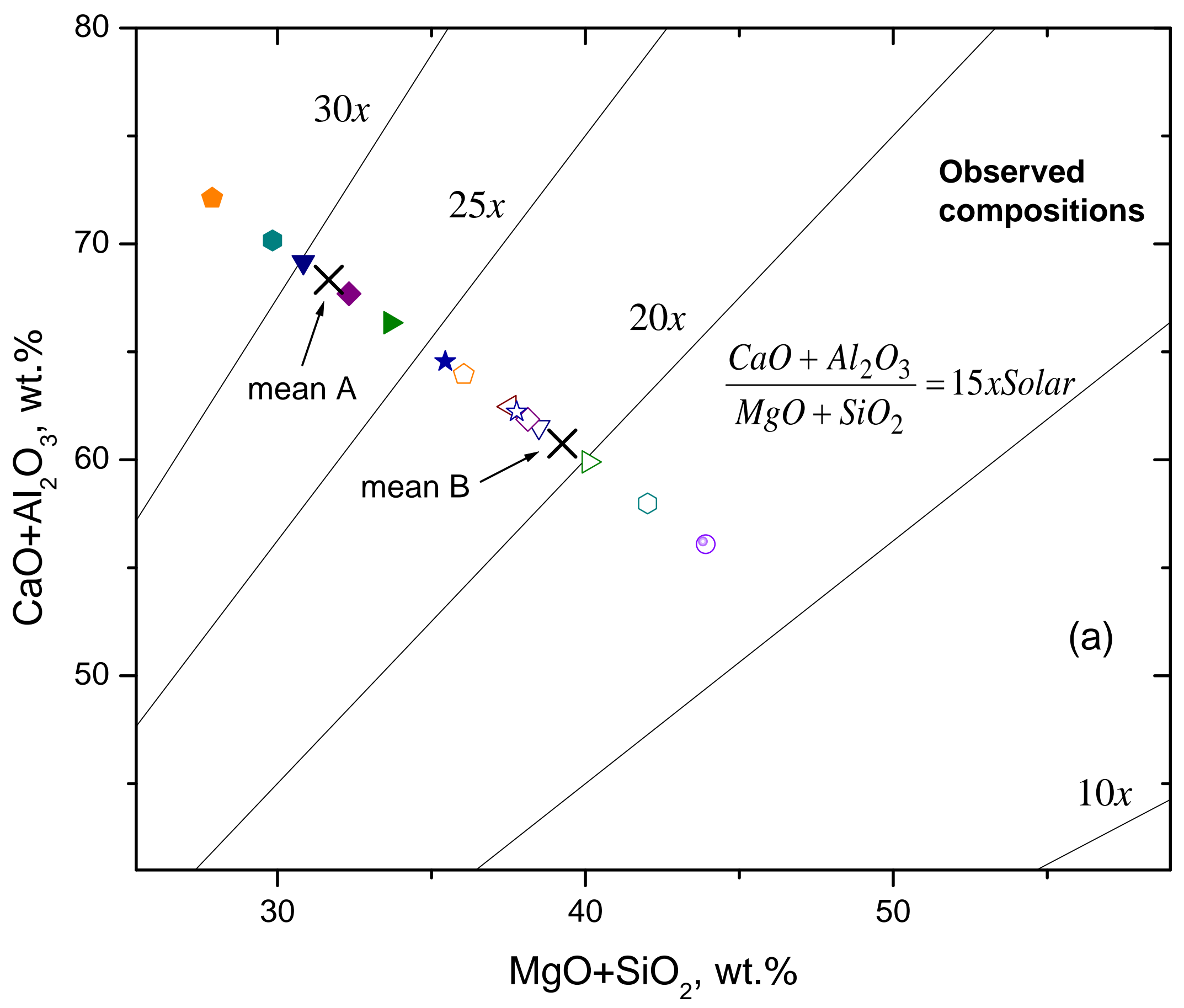




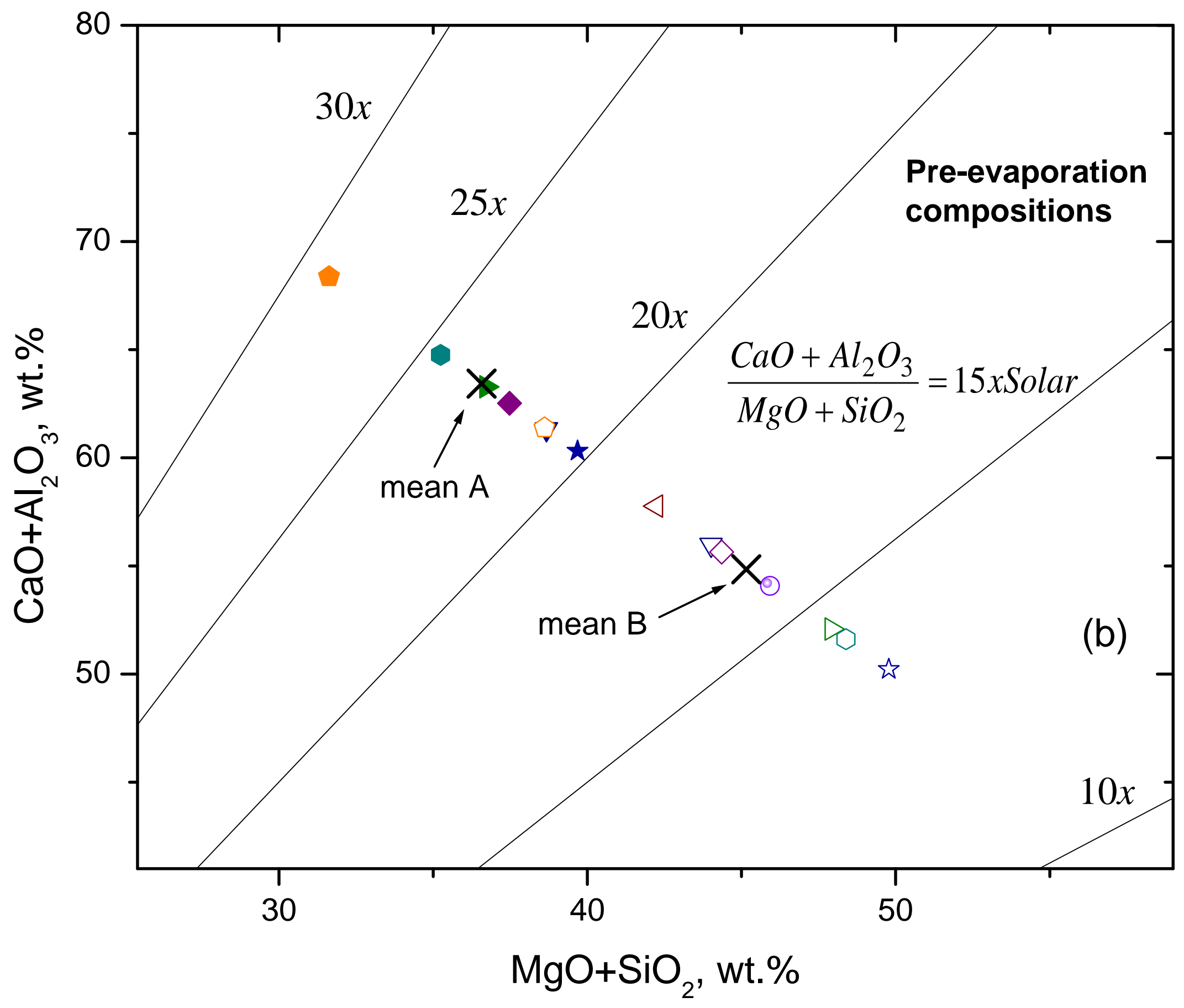

12 